\title{
Fundamental differences in the radio properties of red and blue quasars: enhanced compact AGN emission in red quasars
}

\author{
V. A. Fawcett, ${ }^{1 \star}$ D. M. Alexander, ${ }^{1}$ D. J. Rosario,${ }^{1}$ L. Klindt, ${ }^{1}$ S. Fotopoulou, ${ }^{1,2}$ \\ E. Lusso, ${ }^{3,4}$ L. K. Morabito, ${ }^{1}$ \& G. Calistro Rivera ${ }^{5}$ \\ ${ }^{1}$ Centre for Extragalactic Astronomy, Department of Physics, Durham University, DH1 3LE, UK \\ ${ }^{2}$ HH Wills Physics Laboratory, University of Bristol, Tyndall Avenue, Bristol, BS8 1TL, UK \\ ${ }^{3}$ Dipartimento di Fisica e Astronomia, Università di Firenze, via G. Sansone 1, I-50019 Sesto Fiorentino, Firenze, Italy \\ ${ }^{4}$ Osservatorio Astrofisico di Arcetri, Largo Enrico Fermi 5, I-50125 Firenze, Italy \\ ${ }^{5}$ European Southern Observatory, Karl-Schwarzchild-Strasse 2, 85748, Garching bei Mnchen, Germany
}

10 April 2020

\begin{abstract}
We have recently used the Faint Images of the Radio Sky at Twenty-centimeters (FIRST) survey to show that red quasars have fundamentally different radio properties to typical blue quasars: a significant (factor $\approx 3$ ) enhancement in the radio-detection fraction, which arises from systems around the radio-quiet threshold with compact $\left(<5^{\prime \prime}\right)$ radio morphologies. To gain greater insight into these physical differences, here we use the DR14 Sloan Digital Sky Survey (SDSS) and more sensitive, higher resolution radio data from the Very Large Array (VLA) Stripe 82 (S82) and VLA-COSMOS $3 \mathrm{GHz}(\mathrm{C} 3 \mathrm{GHz})$ surveys. With the S82 data, we perform morphological analyses at a resolution and depth three times that of the FIRST radio survey, and confirm an enhancement in radio-faint and compact red quasars over typical quasars; we now also find tentative evidence for an enhancement in red quasars with slightly extended radio structures $(16-43 \mathrm{kpc}$ at $z=1.5)$. These analyses are complemented by $\mathrm{C} 3 \mathrm{GHz}$, which is deep enough to detect radio emission from star-formation processes. From our data we find that the radio enhancement from red quasars is due to AGN activity on compact scales $(\lesssim 43 \mathrm{kpc})$ for radio-intermediate-radio-quiet sources $(-5<\mathcal{R}<-3.4$, where $\left.\mathcal{R}=L_{1.4 \mathrm{GHz}} / L_{6 \mu \mathrm{m}}\right)$, which decreases at $\mathcal{R}<-5$ as the radio emission from star-formation starts to dilute the AGN component. Overall our results argue against a simple orientation scenario and are consistent with red quasars representing a younger, earlier phase in the overall evolution of quasars.
\end{abstract}

Key words: galaxies: active - galaxies: evolution - galaxies: jets - quasars: general - quasars: supermassive black holes - radio continuum: galaxies

\section{INTRODUCTION}

Quasi-stellar objects (QSOs), also known as quasars, are the most powerful class of Active Galactic Nuclei (AGN). Their extremely high bolometric luminosities (up to $10^{47-48} \mathrm{erg} \mathrm{s}^{-1}$ ) are now known to be caused by accretion onto a supermassive black hole (SMBH; $10^{8}-10^{9} \mathrm{M}_{\odot}$ ) near the Eddington limit which places them as some of the most luminous objects in the Universe.

Due to the unobscured view of the SMBH accretion disc, which peaks in the ultra-violet (UV), the majority of Type 1 QSOs have very blue optical colours. However, there is a small but significant subset with redder optical-infrared colours (coined as "red QSOs"). Although red QSOs have been well studied in the literature (Webster et al. 1995; Serjeant \& Rawlings 1996; Kim \& Elvis 1999;

^ E-mail: victoria.fawcett@durham.ac.uk
Richards et al. 2003; Glikman et al. 2007; Georgakakis et al. 2009; Urrutia et al. 2009; Banerji et al. 2012; Glikman et al. 2012; Kim \& Im 2018; Klindt et al. 2019), their exact nature remains unclear.

The origin of the red colours has been widely debated: for the majority of red QSOs the reddening appears to be due to extinction by dust e.g. (Webster et al. 1995; Glikman et al. 2007; Klindt et al. 2019), although a red synchrotron component or stellar contamination from the host-galaxy may also contribute in some systems (Whiting et al. 2001). However, it is unclear whether this dust is on host-galaxy or nuclear scales (Hickox \& Alexander 2018). The latter could just be a consequence of the AGN orientation model (Urry \& Padovani 1995), with red QSOs representing a grazing incidence viewing angle through the dusty torus. An alternative hypothesis is that red QSOs represent a rapid evolutionary phase that links obscured star-formation with AGN activity (Hopkins et al. 2006, 2008; Alexander \& Hickox 2012). In this context, merger-driven 
AGN activity is thought to drive a starburst phase, resulting in obscuration by dust in the early stages which is then blown out through AGN-driven outflows (commonly referred to as "AGN feedback"), eventually resulting in an unobscured AGN (within the context of our study, a blue unobscured QSO). Some studies claim to see merger induced activity in red QSOs (Urrutia et al. 2008; Urrutia et al. 2012; Glikman et al. 2015). However, it is less clear that red QSOs systematically show an enhancement in merger signatures when compared to typical QSOs (e.g., Zakamska et al. 2019).

Taking a novel approach to uniformly define their QSO samples, Klindt et al. (2019) used Sloan Digital Sky Survey (SDSS) DR7 data (Schneider et al. 2010) to optically select the top, bottom and middle 10 percentiles of the redshift-dependent observed optical colour distribution to create a red, blue and control ("typical") QSO sample, respectively. They found a factor $\approx 3$ larger radio detection rate in the red QSO sample making use of Faint Images of the Radio Sky at Twenty-centimeters (FIRST; Becker et al. 1995) data, compared to both the blue and control QSOs. The radio-detection enhancement was driven by red QSOs with compact radio morphologies ( $<5^{\prime \prime} ;<43 \mathrm{kpc}$ at $\left.z=1.5\right)$ and luminosities placing them around the radio-loud/radio-quiet threshold. These results rule out the simple orientation model, suggesting differences in the "environment" between red QSOs and typical QSOs which may be driven within the evolutionary sequence ${ }^{1}$.

QSOs exhibit a wide range of radio morphologies, with some that display large-scale jets and lobes that can extend over Mpc scales (e.g. Krishna et al. 1998). The main classifications can be split into compact, where the radio emission is spatially unresolved on scales of a few arcseconds, and extended morphologies. The extended category includes the spectacular Fanaroff-Riley (FR) type I and II (Fanaroff \& Riley 1974) systems, as well as resolved diffuse emission. This binary distinction is simplistic, with sources that can show small-scale jets (few pc to tens of kpc), such as compact steep spectrum (CSS; Fanti et al. 1990, 1995) or gigahertz-peaked spectrum (GPS; Stanghellini et al. 1998; O’Dea et al. 1991) sources. Recent studies have shown that FRII sources also have low-powered jets with low radio luminosities (Mingo et al. 2019).

In this work we use deep and high-resolution radio data to further investigate the origins of the difference in the radio properties between red QSOs and typical QSOs down to kpc scales and significantly fainter radio fluxes than explored in Klindt et al. (2019). We use two different high-resolution Very Large Array (VLA) radio surveys: VLA Stripe 82 (Hodge et al. 2011) and VLA-COSMOS $3 \mathrm{GHz}$ (Smolčić et al. 2017a), which are $\sim 3$ and 38 times deeper than the FIRST survey, respectively. We select our red QSO sample from the SDSS DR14 catalogue (Pâris et al. 2018) and follow a similar approach to Klindt et al. (2019), using the SDSS colours to define red and control QSOs (see Section 2). In Section 3.1 we explore the radio enhancement in red QSOs down to lower radio fluxes than in Klindt et al. (2019) and test how it varies across the radio-loudness plane. In Section 3.2 we use median stacking to explore the radio properties of the undetected population, pushing far below the survey flux threshold and in Section 3.3 we focus on the morphological properties of our red QSO sample, probing finer scales than in Klindt et al. (2019). In Section 4.1 we use the deeper COSMOS data to constrain the star-formation contribution to the radio emission from the QSOs and in Section 4.2 we comment

\footnotetext{
1 In this work we use the term "environment" to indicate the environment on anything from nuclear and host-galaxy scales to much larger physical scales (i.e., the dark matter halo).
}

on the overall fraction of QSOs that have radio emission potentially dominated by AGN and non-AGN processes. Our results add weight to the emerging picture that red QSOs are fundamentally different to typical QSOs. Throughout our work we adopt a flat $\Lambda$-cosmology with $H_{0}=70 \mathrm{kms}^{-1} \mathrm{Mpc}^{-1}, \Omega_{\mathrm{M}}=0.3$ and $\Omega_{\Lambda}=0.7$.

\section{DATA SETS AND METHODS}

In this paper we explore the high-resolution radio properties of SDSS optically selected QSOs at $0.2<z<2.4$. The overall quasar selection process used is similar to that adopted in Klindt et al. (2019). However, in our work we now select QSOs from the SDSS DR14 Quasar Catalogue (Pâris et al. 2018), as opposed to Klindt et al. (2019) who used the SDSS DR7 catalogue (Schneider et al. 2010 ), which provides a factor $\approx 5$ improvement in sample size, as well as going almost two magnitudes deeper in the optical. In this section we describe our selection strategy, the radio surveys utilised, and the key measurements extracted from the multi-wavelength data in the SDSS. Fig. 1 shows a schematic representation of the sample selection, which we describe in more detail in the following subsections.

\subsection{Parent sample: optical and mid-infrared data}

\subsubsection{SDSS DR14 Quasar Catalogue}

The SDSS DR14 Quasar Catalogue (Pâris et al. 2018) contains 526356 spectroscopically selected QSOs with luminosities $M_{i}[z=2]<-20.5$, out to redshifts around $z=7$. The survey covers a region of $9376 \mathrm{deg}^{2}$ and consists of various different targeting campaigns. The catalogue includes previous spectroscopicallyconfirmed QSOs from the SDSS-I and II Legacy surveys (York et al. 2000) with QSOs targeted by the Baryon Oscillation Spectroscopic Survey (BOSS; Dawson et al. 2013) in SDSS-III (Eisenstein et al. 2011) and the extended Baryon Oscillation Spectroscopic Survey (eBOSS; Dawson et al. 2016) in SDSS-IV. As explained in Section 2.3.1, we removed the eBOSS-targeted QSOs from our final samples due to differences in the source densities of the two Stripe 82 regions.

The Pâris et al. (2018) quasar catalogue provides spectroscopic redshifts based on different estimators; in this work we use the most robust of these estimates (listed as $Z$ in the catalogue). The SDSS five-band optical photometry (ugriz) is also utilised, corrected by the associated band-dependent Galactic extinction measurements.

\subsubsection{Mid-infrared counterparts: matching to WISE}

We matched the SDSS DR14 quasar sample to mid-infrared (MIR) counterparts using the Wide-Field Infrared Survey Explorer (WISE; Wright et al. 2010), an all-sky survey which provides photometry in four bands (3.4, 4.6, 12 and $22 \mu \mathrm{m})$. The emission from the QSO accretion disc is expected to be absorbed and reradiated by hot dust at the inner edge of the torus, leading to an infrared (IR) excess that peaks at MIR wavelengths. Therefore the MIR emission is a useful, extinction-insensitive discriminant of QSOs (e.g. Stern et al. 2005; Lacy et al. 2005; Stern et al. 2012; Assef et al. 2013), as well as a measure of their intrinsic luminosity; for example the commonly used rest-frame $6 \mu \mathrm{m}$ luminosity $\left(L_{6 u \mathrm{~m}}\right)$.

We used the NASA/IPAC query engine to match SDSS DR14 QSOs to the All-Sky WISE Source Catalogue (ALL-WISE) adopting a $2 . \prime 7$ search radius, which ensured a $95.5 \%$ positional certainty 


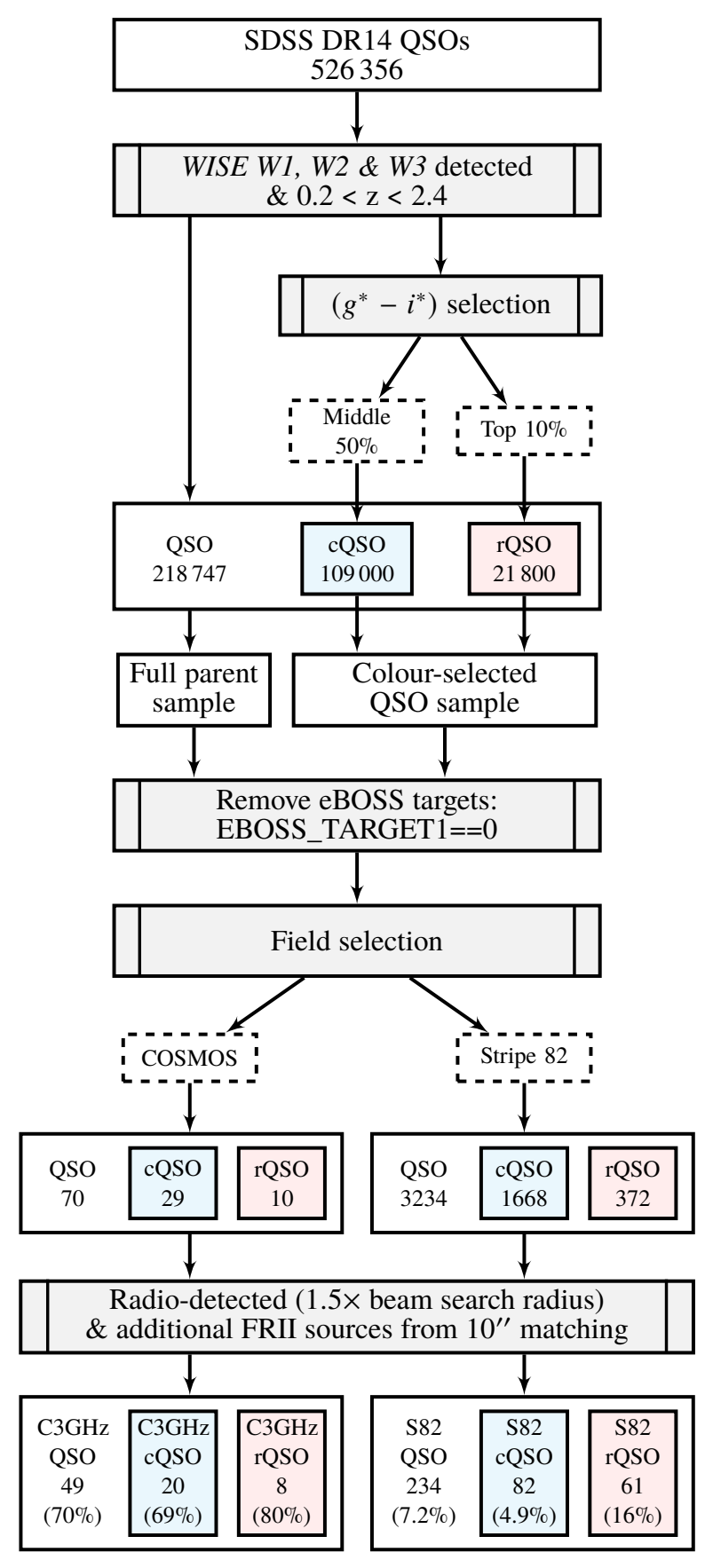

Figure 1. A schematic diagram illustrating the selection process used to obtain our radio-detected samples, starting from the full SDSS DR14 quasar catalogue. We selected QSOs with redshifts $0.2<z<2.4$ and matched to WISE using the NASA/IPAC query engine with a 2 .' 7 search radius, requiring a SNR $>2$ in $W 1, W 2$ and $W 3$. Our QSO colour selection was then applied to identify rQSOs and cQSOs as the top $10 \%$ and middle $50 \%$ of the redshift dependent observed $\left(g^{*}-i^{*}\right)$ colour distribution, respectively. We focus our study on the VLA-COSMOS $3 \mathrm{GHz}$ and $1.4 \mathrm{GHz}$ Stripe 82 surveys, matching the QSOs in these fields to the radio catalogues using a search radius 1.5 times the survey beam size; we additionally include FRIIlike sources with weak radio cores but bright lobes that are missed with this approach using a $10^{\prime \prime}$ search radius. Due to the discrepancy in the QSO targeting between the two regions of Stripe 82, eBOSS-targeted QSOs were removed before field selection (see Appendix A).

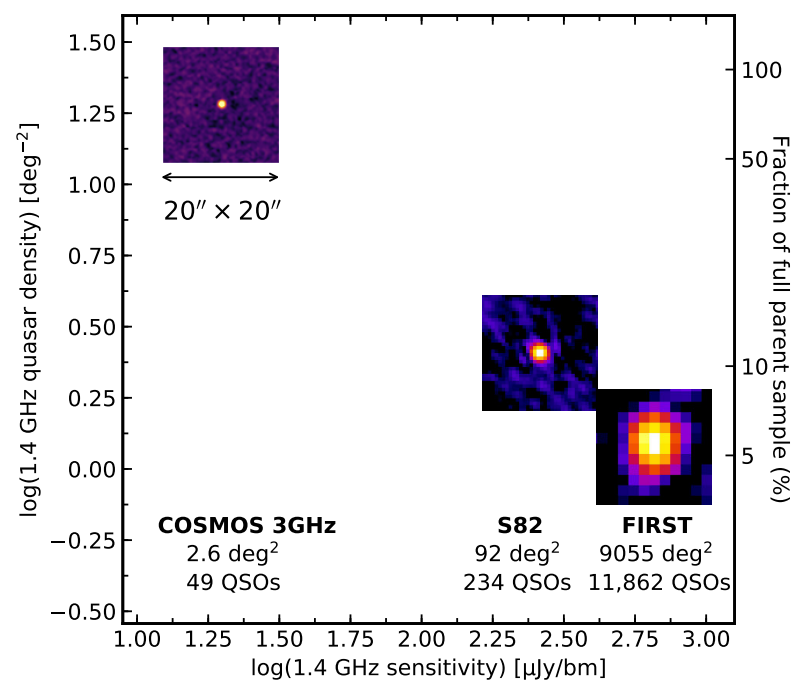

Figure 2. Radio-detected parent sample QSO source density versus $5 \sigma$ $1.4 \mathrm{GHz}$ sensitivity for the three radio surveys considered in this paper. For $\mathrm{C} 3 \mathrm{GHz}$, the $1.4 \mathrm{GHz}$ sensitivity was calculated from the $3 \mathrm{GHz}$ sensitivity limit assuming a uniform spectral slope of $\alpha=-0.7$. The $20^{\prime \prime} \times 20^{\prime \prime}$ thumbnails give a visual illustration of the resolution for a compact (i.e., unresolved) radio source in the different radio surveys; the number of radiodetected $\mathrm{C} 3 \mathrm{GHz}, \mathrm{S} 82$ and FIRST parent sample QSOs is shown underneath (see Table 1). The source density as a fraction of the full parent sample is displayed on the right-hand axis as a reference.

(Lake et al. 2011), and required a detection with a signal-to-noise ratio (SNR) of greater than 2 in the WISE W1,W2 and W3 bands. As in Klindt et al. (2019), we only selected QSOs with redshifts between $0.2<z<2.4$ : the higher redshift cut ensures there is no contamination from the Lyman break in the $g^{*}$ band and the lower redshift cut ensures we only consider luminous QSOs in our sample.

The need for a WISE detection in the three bands and the restriction of $0.2<z<2.4$ reduced the number of QSOs to 218747 , the full parent sample; see Fig. 1. To create the C3GHz and S82 parent samples (70 and 3234 QSOs, respectively; see Table 1 and Fig. 1), we have restricted the full parent sample to the regions covered by these two radio surveys, using the average root mean square (RMS) mosaics (see Sections 2.2.1 and 2.2.2). Using WISE, we computed the rest-frame $6 \mu \mathrm{m}$ flux with a log-linear interpolation (or extrapolation) of the $W 2$ and $W 3$ bands following the approach outlined in Klindt et al. (2019). The flux was then converted into a luminosity $\left(L_{6 \mu \mathrm{m}}\right)$ using the cosmological luminosity distance.

\subsection{Radio data}

The main focus of this paper is to understand the differences in the radio properties between red and typical QSOs. We used data from two high spatial resolution VLA radio surveys: VLA Stripe 82 (S82; Hodge et al. 2011) and VLA-COSMOS 3 GHz (C3GHz; Smolčić et al. 2017a), which we describe in more detail below. To better place these data into context with our earlier Klindt et al. (2019) paper, we also describe the VLA FIRST survey used in that work.

Fig. 2 compares the radio source density of the $\mathrm{C} 3 \mathrm{GHz}, \mathrm{S} 82$ and FIRST parent sample QSOs matched to the three different radio survey catalogues, using a search radius 1.5 times the survey beam size for S82 and C3GHz, and 10" for FIRST (to be consistent with Klindt et al. 2019), as a function of the $5 \sigma$ sensitivity limit for each survey; $70 \%$ of the parent QSOs are detected in $\mathrm{C} 3 \mathrm{GHz}$, an order of 
magnitude greater than in FIRST. The cutouts illustrate a compact (i.e., unresolved) radio source at the different survey resolutions.

\subsubsection{VLA Stripe 82 (S82)}

Stripe 82 is a $\sim 300 \mathrm{deg}^{2}$ equatorial field that has been imaged multiple times by SDSS (Jiang et al. 2014). It spans a right ascension of $\alpha=-50^{\circ}$ to $+59^{\circ}$ and a declination of $\delta=-1.25^{\circ}$ to $1.25^{\circ}$. The highresolution radio survey, S82 (Hodge et al. 2011), covers $\sim 92 \mathrm{deg}^{2}$ and has a 1.' 8 spatial resolution at $1.4 \mathrm{GHz}$, taken primarily in the A-configuration, to a sensitivity roughly three times below that of FIRST. We note that Heywood et al. (2016) provide a similar survey using the VLA hybrid $\mathrm{CnB}$ configuration, but at a lower resolution $\left(16^{\prime \prime} \times 10^{\prime \prime}\right)$ and shallower depth; this survey recovers some lost flux from sources in Stripe 82 with diffuse radio lobes, but results in a lower overall number of sources. As a key focus of our study is high spatial resolution, we have only used the Hodge et al. (2011) radio survey.

In the catalogue provided by Hodge et al. (2011), peak flux densities $\left(F_{\text {peak }}\right)$ are derived by fitting an elliptical Gaussian model to the source. The fitted major axis used for physical size calculations are derived from the elliptical Gaussian model, fitted before deconvolution of the circular clean beam. Matching to the FIRST catalogue, Hodge et al. (2011) recover over 97\% of the FIRSTdetected QSOs.

\subsubsection{VLA-COSMOS $3 \mathrm{GHz}(\mathrm{C} 3 \mathrm{GHz})$}

The C3GHz survey spans $2.6 \mathrm{deg}^{2}$ at $3 \mathrm{GHz}$, centred on the COSMOS field $(\mathrm{RA}=10: 00: 28.6, \mathrm{Dec}=+02: 12: 21.0)$ at a 0.75 spatial resolution in the $\mathrm{A}+\mathrm{C}$ configuration (Smolčić et al. 2017a). At its median depth $(\sim 7 \mu \mathrm{Jy}$ at $5 \sigma), \mathrm{C} 3 \mathrm{GHz}$ can detect a source that is equivalently 13 times deeper than the S82 sensitivity limit at $1.4 \mathrm{GHz}$, assuming a spectral index of $\alpha=-0.7\left(S_{v} \propto v^{\alpha} ; \sim 38\right.$ times deeper than FIRST); C3GHz is currently the largest and deepest radio continuum survey at such a high-resolution (Smolčić et al. 2017a). In the catalogue provided by Smolčić et al. (2017a), $F_{\text {peak }}$ is measured by fitting a two-dimensional parabola around the brightest pixel. Using a Monte Carlo method, they derived a source completeness of $55 \%$ up to $20 \mu \mathrm{Jy}$, which rises to $94 \%$ above $40 \mu \mathrm{Jy}$.

The COSMOS field has also been observed at $1.4 \mathrm{GHz}$ over $2 \mathrm{deg}^{2}$ in an earlier VLA radio survey (Schinnerer et al. 2007). This survey has a resolution of 1 .' $^{\prime} \times 11^{\prime \prime}$. 4 and an RMS of $\sim 10.5 \mu \mathrm{Jy} / \mathrm{bm}$ $(\sim 5$ times deeper than S82) and is also utilised in this paper when available, for comparison with the $\mathrm{S} 82$ data.

\subsubsection{VLA FIRST}

The FIRST radio survey covers $9055 \mathrm{deg}^{2}$ of the North Galactic Cap and Equatorial Strip in the SDSS region. FIRST has a $5^{\prime \prime}$ resolution at $1.4 \mathrm{GHz}$ taken primarily in the VLA B-configuration. The catalogue (Becker et al. 1995) contains 946000 sources with a typical RMS of $0.15 \mathrm{mJy} ; 30 \%$ of the FIRST sources have optical counterparts in the SDSS.

\subsection{Red and control QSO samples}

One of the challenges in comparing between different red QSO studies is the variety of selection methods used to obtain a red QSO sample. In this paper we take the same approach as Klindt et al. (2019), distinguishing between red and typical QSOs based

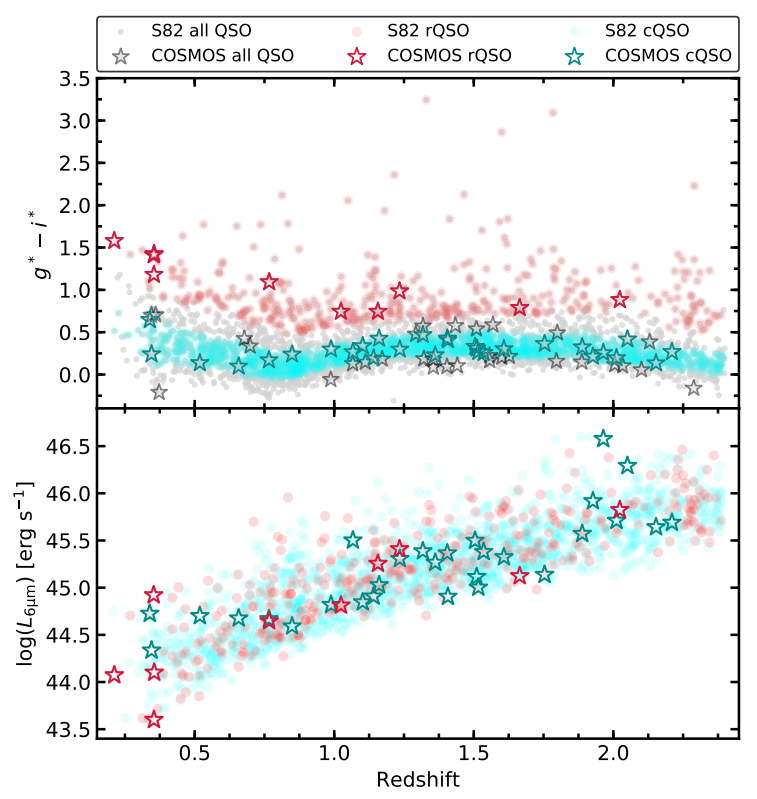

Figure 3. Sample distribution comparison of observed $g^{*}-i^{*}$ colour (top) and rest-frame $L_{6 \mu \mathrm{m}}$ luminosity (bottom) versus redshift. The filled circles represent S82 QSOs and the open stars represent the $\mathrm{C} 3 \mathrm{GHz}$ QSOs: the rQSOs and cQSOs are plotted in red and cyan, respectively. The grey points in the top plot illustrate the colours of the parent sample DR14-WISE detected QSOs within the two fields, with the eBOSS-selected sources removed (Stripe 82: 3234; COSMOS: 70). The top plot shows our redshift dependent colour-selection approach and the bottom plot shows that the range in $L_{6 \mu \mathrm{m}}$ and redshift for the two samples are consistent.

on SDSS optical photometry in a redshift dependent manner. Selecting a control sample in a consistent way allows us to robustly identify differences with respect to the red QSO population, in contrast to most other studies which use a separate control sample (often with a different selection approach and wavebands) for their comparison. Our selection is quantitatively different to that in Klindt et al. (2019), who defined their control QSOs as the middle 10th percentile of the $g^{*}-i^{*}$ distribution, in addition to a blue QSO sample (the bottom 10th percentile). We do not define a blue QSO sample here since Klindt et al. (2019) demonstrated that there are no significant differences between the blue and control QSO samples and consequently we also defined a broader control QSO sample to improve the source statistics in our comparisons with the red QSOs. Our companion study by Rosario et al. (2020) also employs a similar colour selection.

We note that by only using optical photometry we expect our colour selection to result in red QSOs with extinction values of $A_{V} \sim 0.1-0.5 \mathrm{mag}$ (see Section 2.2.3 in Klindt et al. 2019), missing the reddest systems (e.g. Glikman et al. 2012; Banerji et al. 2015 also exploit near-IR photometry to select QSOs with even redder colours); however, we highlight that a key benefit of our approach is the consistent selection of both red and control QSOs.

\subsubsection{Colour-selected samples}

To define our sample of colour-selected QSOs we used the $g^{*}$ $(4770 \AA)$ and $i^{*}(7625 \AA)$ band extinction-corrected photometry. Our red and control QSOs (hereafter, rQSOs and cQSOs) were selected above the top 90th percentile and within the middle 50th percentile of the observed SDSS $g^{*}-i^{*}$ distribution, respectively; 
Table 1. The number of parent QSOs, rQSOs and cQSOs, and their radiodetection fraction, for our two samples; see Fig. 1 for selection process and sample numbers. The parent QSOs are selected within the two regions before the colour-selection. The radio-detection enhancement of the rQSOs in comparison to the cQSOs is also shown, where we see a clear enhancement for the S82 sample but only a tentative enhancement in the deeper $\mathrm{C} 3 \mathrm{GHz}$ sample.

\begin{tabular}{ll|llll}
\hline \hline \multicolumn{2}{c|}{ Sample } & Num. & $\begin{array}{l}\text { Radio } \\
\text { detected }\end{array}$ & $\begin{array}{l}\text { Detection } \\
\%\end{array}$ & $\begin{array}{l}\text { rQSO radio } \\
\text { enhancement }\end{array}$ \\
\hline \multirow{3}{*}{ S82 } & Parent & 3234 & 234 & 7.2 & - \\
& rQSOs & 372 & 61 & 16 & $3.3_{-0.5}^{+0.6}$ \\
& cQSOs & 1668 & 82 & 4.9 & - \\
\multirow{3}{*}{$\mathrm{C} 3 \mathrm{GHz}$} & Parent & 70 & 49 & 70 & \\
& rQSOs & 10 & 8 & 80 & $1.2_{-0.3}^{+0.2}$ \\
& cQSOs & 29 & 20 & 69 & \\
\hline \hline
\end{tabular}

the cQSOs therefore represent typical QSOs. In order to produce a redshift sensitive colour sample, the QSOs were sorted by redshift and the $g^{*}-i^{*}$ distribution was binned using contiguous redshift bins consisting of 1000 sources. This produced a sample of 130800 QSOs (21 800 rQSOs and 109000 cQSOs; see Fig. 1). The $g^{*}-i^{*}$ colour distribution of the colour-selected samples is shown in the top panel of Fig. 3.

The SDSS-IV eBOSS survey (see Section 2.1.1) only covered the Eastern part of the Stripe 82 (known as East: RA $\lesssim 36 \mathrm{deg}$ ), which leads to a difference in the sky densities of QSOs with respect to the Western part (know as West: RA $\gtrsim 330 \mathrm{deg}$ ), as well as a difference in the QSO redshift distributions. Therefore, we excluded the eBOSS-targeted QSOs from the colour-selected sample and S82 parent sample (see Fig. 1; we note there are no eBOSS-targeted QSOs in COSMOS). This meant the two regions of Stripe 82 can be combined in a consistent way (see Appendix A for more details).

For our study here, the colour-selected QSO sample (with eBOSS-targeted QSOs removed) was then restricted to the S82 and $\mathrm{C} 3 \mathrm{GHz}$ survey regions. For S82, we defined the areas using the online catalogue matching service provided by Hodge et al. (2011). This provided non-zero local RMS values for detected and undetected QSOs within the survey region, which resulted in 2040 colour-selected QSOs within S82 (1668 cQSOs and 372 rQSOs). For C3GHz the RMS mosaic was used to only select QSOs within the radio-observed region of COSMOS, which resulted in 39 colourselected QSOs (29 cQSOs and $10 \mathrm{rQSOs})^{2}$. Selecting the colours before restricting to the two fields ensured that the two colour-selected samples are representative of the entire DR14 QSO population.

The bottom panel of Fig. 3 displays the $L_{6 \mu \mathrm{m}}-z$ distributions for the colour-selected QSO sample in the $\mathrm{S} 82$ and $\mathrm{C} 3 \mathrm{GHz}$ regions. Applying the two-sided Kolmogorov-Smirnov test for the S82 and $\mathrm{C} 3 \mathrm{GHz}$ rQSO and cQSO redshift distributions separately, we cannot rule out that the two samples are drawn from the same parent distribution at a $\sim 20 \%$ and $10 \%$ significance, respectively, which shows that the redshift distribution of the red and control samples are broadly consistent with each other.

\subsubsection{Final radio-detected samples: $\mathrm{S} 82$ and $\mathrm{C} 3 \mathrm{GHz}$}

The colour-selected QSO samples were matched to the highresolution radio catalogues using a search radius of 1.5 times that of the beam size (see Sections 2.2.1 and 2.2.2). This resulted in an

2 The VLA-COSMOS $3 \mathrm{GHz}$ RMS mosaic is available online (http://jvla-cosmos.phy.hr/Home.html).
Table 2. Percentage of our colour-selected and radio-detected final samples that lie outside the AGN wedge $(W 1-W 2$ vs $W 2-W 3)$ from Mateos et al. (2012). We find that the majority of QSOs in our two samples have WISE colours consistent with AGN.

\begin{tabular}{l|cc|cc}
\hline \hline \multicolumn{5}{c}{ Outside AGN Wedge } \\
\hline & Stripe 82 & \multicolumn{2}{c}{ COSMOS } \\
& Num. & $\%$ & Num. & $\%$ \\
\hline \multicolumn{5}{c}{ Colour-selected } \\
\hline cQSO & $37 / 372$ & $9.9 \%$ & $1 / 10$ & $10 \%$ \\
\hline \multicolumn{5}{c}{ Radio-detected } \\
\hline rQSO & $117 / 1668$ & $7.0 \%$ & $1 / 29$ & $3.4 \%$ \\
cQSO & $4 / 61$ & $6.6 \%$ & $0 / 8$ & $0 \%$ \\
\hline \hline
\end{tabular}

initial sample of 139 S82-detected QSOs (60 rQSOs and 79 cQSOs) and $28 \mathrm{C} 3 \mathrm{GHz}$-detected QSOs (8 rQSOs and $20 \mathrm{cQSOs}$ ). Based on the analysis of $\mathrm{Lu}$ et al. (2007) (applied to the deeper depths of these surveys), we expect a false association rate of $\sim 0.2 \%$ and $0.6 \%$ for $\mathrm{C} 3 \mathrm{GHz}$ and $\mathrm{S} 82$, respectively. To take into account rarer FRII-like sources, whose cores may be too faint to be detected and whose radio lobes can extend beyond our search radius, we matched again using a $10^{\prime \prime}$ search radius and summed the radio flux from all sources within that radius. This produced an extra 12 potential matches for the S82 data, and 1 for $\mathrm{C} 3 \mathrm{GHz}$. These sources were visually inspected and only the FRII-like sources were added to our samples (S82: 4; C3GHz: 0, see Fig. 4), with the remaining sources discounted as spurious matches (see Fig. B1). This gave a final sample of 143 S82-detected QSOs (61 rQSOs and 82 cQSOs) and 28 C3GHz-detected QSOs (8 rQSOs and 20 cQSOs); see Fig. 1. The C3GHz and S82 parent samples (70 and 3234 QSOs, respectively) were also matched to the respective radio surveys following the same method as the colour-selected samples, resulting in 49 and 234 radio-detected $\mathrm{C} 3 \mathrm{GHz}$ and $\mathrm{S} 82$ parent sample QSOs, respectively. Table 1 displays the number of QSOs and radio-detection fraction for the parent QSOs, rQSOs and cQSOs in the two surveys, and the overall selection process is shown in Fig. 1.

To verify that the MIR emission of our final samples is dominated by the AGN, we explored the WISE $W 1-W 2$ versus $W 2-W 3$ colour-colour space, in which Mateos et al. (2012) defined a region used to reliably select luminous AGN (the "AGN wedge"). The percentage of our colour-selected and radio-detected samples that lie outside the AGN wedge are given in Table. 2. This shows that the majority of the QSOs ( $\sim 90-100 \%$ for COSMOS and $\sim 90-95 \%$ for Stripe 82 ) lie within the wedge. The outliers are predominately the low luminosity sources at all redshifts, with the majority at either the lowest or highest end of our redshift range. The low luminosity end may have a significant level of host-galaxy contamination, as also suggested in Klindt et al. (2019). For the high redshift end $(z>2)$, it is known that the AGN wedge becomes less reliable at selecting AGN (see Figure 5 in Mateos et al. 2012).

The rest-frame $1.4 \mathrm{GHz}$ luminosities were calculated for $\mathrm{S} 82$ by assuming a uniform spectral slope of $\alpha=-0.7$, following Alexander et al. $(2003)^{3}$. For the $\mathrm{C} 3 \mathrm{GHz}$ sample, the $1.4 \mathrm{GHz}$ fluxes were taken from the VLA-COSMOS 1.4 GHz survey (Schinnerer et al. 2007) when available, and calculated from the $3 \mathrm{GHz}$ fluxes assuming a uniform spectral slope of $\alpha=-0.7$ otherwise. If $1.4 \mathrm{GHz}$ fluxes were available, the rest-frame $1.4 \mathrm{GHz}$ luminosities were calculated using the measured spectral slope between 1.4 and $3 \mathrm{GHz}$.

\footnotetext{
3 In this paper we define the radio spectral slope $(\alpha)$ as $S_{v} \propto v^{\alpha}$.
} 
FRII rQSOS

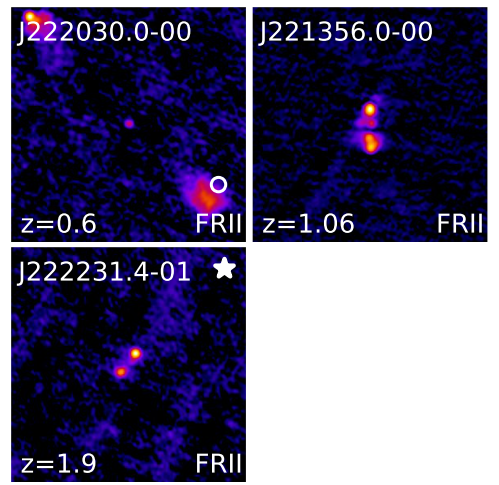

FRII cQSOS

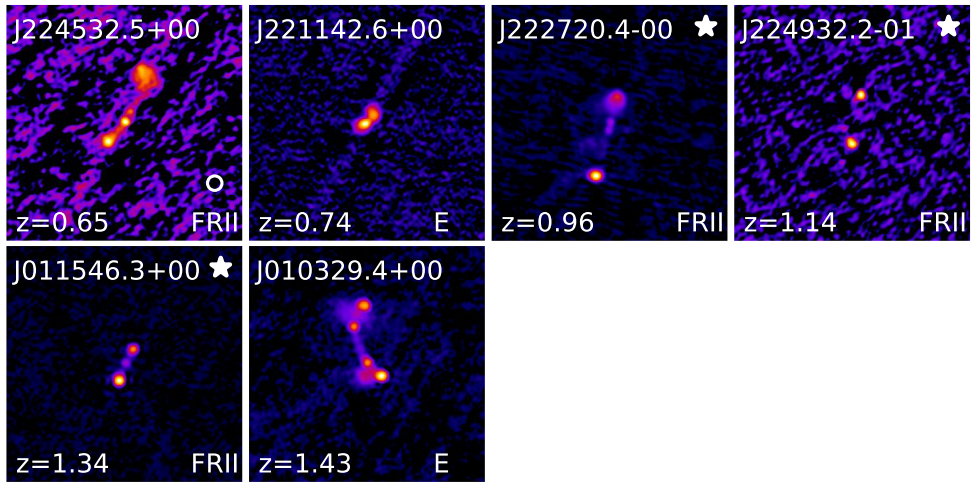

Figure 4. Thumbnails (1.5 × 1.5) using data from Hodge et al. (2011) of S82 FRII-like rQSOs (left) and cQSOs (right). The morphology classification (see Section 3.3) from visually inspecting the FIRST VLA data is shown in the bottom right of the image; FRII (FRII-like) or E (extended). The redshift is shown in the bottom left corner and the VLA source name is shown at the top left corner. The star in the top right corner indicates the 4 FRII sources included from the wider $10^{\prime \prime}$ search radius. The white circle in the first thumbnail illustrates the 1 ." 8 beam size of the VLA data.

\subsection{Visual assessment of radio morphologies}

To determine the radio morphologies of the colour-selected QSOs we took the same approach as that used in Klindt et al. (2019). We created 1.5 square cutouts for all sources, and visually inspected them in random order, blind to whether they were part of the rQSO or cQSO sub-samples so as not to bias our judgement. For the S82 analysis, the FIRST images were also inspected to obtain morphologies using both the S82 data and the FIRST data, which could then be used to explore the change in morphology when using higher resolution and deeper data (see Appendix C). Here we use similar morphology classes to Klindt et al. (2019), where a source can be defined as compact (unresolved; i.e., point-like), extended (single sources with extended emission), FRII-like (double lobed systems with $F_{\text {peak,lobe }}>F_{\text {peak,core }}$ ) or faint; $F_{\text {peak }}<15 \sigma$, where $\sigma$ is the typical RMS of the respective survey (S82: $F_{\text {peak }}<1$ mJy; FIRST: $F_{\text {peak }}<3$ mJy; C3GHz: $\left.F_{\text {peak }}<35 \mu \mathrm{Jy}\right)$. In our study, classic FRI sources $\left(F_{\text {peak,lobe }}<F_{\text {peak,core }}\right)$ are included within the extended category and the compact-FRII class adopted in Klindt et al. (2019) is combined within the FRII-like category due to the rarity of these systems.

\subsection{Median stacking procedure}

Stacking is a method widely used to analyse the average properties of source populations that lie below the detection limit, with most previous studies exploring radio stacking using the FIRST radio data (White et al. 2007; Hodge et al. 2008, 2009; Kratzer \& Richards 2015). We undertook a stacking analysis of the $S 82$ and $C 3 \mathrm{GHz}$ radio data to probe down to $\mu \mathrm{Jy}$ fluxes to assess the average radio properties of red and control QSOs.

We started with a sample of $1897 \mathrm{~S} 82$ radio-undetected QSOs (311 rQSOs, 1586 cQSOs) and $11 \mathrm{C} 3 \mathrm{GHz}$ radio-undetected QSOs ( 2 rQSOs, 9 cQSOs). After removing images with only partial radio coverage, we median stacked a final sample of 290 and 1532 S82undetected rQSOs and cQSOs, respectively, and 2 and $9 \mathrm{C} 3 \mathrm{GHz}-$ undetected rQSOs and cQSOs, respectively. The samples were also stacked in four contiguous redshift bins. Peak flux measurements were obtained by fitting a two-dimensional Gaussian to the stacked map and taking its amplitude. Errors were calculated from the standard deviation of the stacked map after masking a central circular region of $10^{\prime \prime}$; we note that the obtained flux values have not been corrected for any CLEAN or snapshot bias and so will contain additional errors of $\sim 10 \%$ (see White et al. 2007).

\section{RESULTS}

The deep and high-resolution radio data in the Stripe 82 and COSMOS fields (see Sections 2.2.1 \& 2.2.2) allows us to search for fundamental differences between red and typical QSOs. In Klindt et al. (2019) we found that the main differences between the radio properties of red and typical QSOs occurred towards the radio-quiet end of the population, therefore our samples can be used to further probe this faint end, providing insight into the origin and scale of the radio emission.

In Sections 3.1 and 3.2 we analyse the radio-faint population; exploring the radio-loudness parameter and stacking the radioundetected populations. In Section 3.3 we compare the radio morphologies of the rQSOs and cQSOs below the FIRST resolution limit, probing smaller scale radio emission.

\subsection{Radio enhancement in red QSOs}

Comparing the radio-detection fraction of rQSOs and cQSOs, we find an overall enhancement in the radio-detection fraction for rQSOs compared to cQSOs of $\approx 3.3$ for the S82 sample (see Table 1$)^{4}$. For $\mathrm{C} 3 \mathrm{GHz}$, within the uncertainties, there is no enhancement which is primarily due to the majority of the QSO population now being detected in the ultra-deep radio data $(69 \%$ for cQSOs and $80 \%$ for rQSOs).

To explore the radio-detection enhancement in more detail we plotted the cumulative detection fractions of the rQSOs and cQSOs, utilising the $\mathrm{C} 3 \mathrm{GHz}, \mathrm{S} 82$ and FIRST data as a function of radio flux; see Fig. 5. We find that rQSOs exhibit a higher radio-detection fraction compared to the cQSOs in all three surveys, although in regions with low source statistics this enhancement is consistent with unity within the uncertainties. Down to the S82 flux limit, this enhancement is broadly constant at a factor of $\approx 2-3$. These results are therefore in quantitative agreement with Klindt et al. (2019)

4 The radio-detection enhancement is calculated by dividing the radiodetection fraction for the rQSOs by that of the cQSOs. 


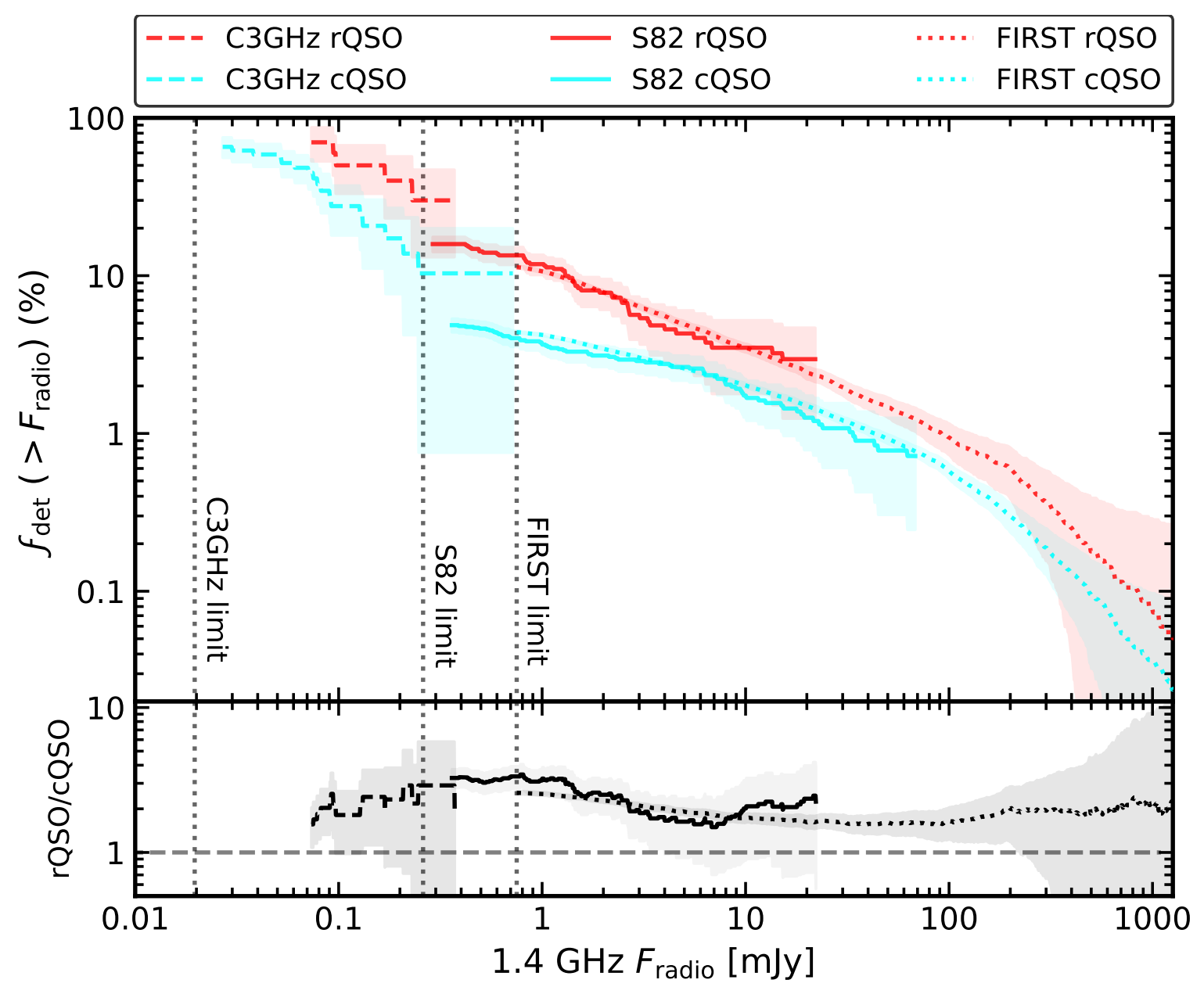

Figure 5. Cumulative radio-detection fractions for the S82, C3GHz and FIRST detected rQSOs (red) and cQSOs (cyan) as a function of radio flux density. The $1.4 \mathrm{GHz}$ flux for the $\mathrm{C} 3 \mathrm{GHz}$ sample was calculated from the $3 \mathrm{GHz}$ flux assuming a uniform spectral slope of $\alpha=-0.7$ (unless $1.4 \mathrm{GHz}$ data was available). The shaded error region was calculated using the method described in Cameron (2011) and corresponds to $1 \sigma$ binomial uncertainties. Cuts were applied at the $5 \sigma$ sensitivity limit of each survey, and data from the bright end was cut when the number of sources became less than 2 (C3GHz) or 10 (S82 \& FIRST) to reduce the widths of the shaded regions; the dotted lines display the $5 \sigma$ flux limits for the three surveys. The bottom panel displays the fractional difference between the radio-detected rQSOs and the radio-detected cQSOs. Across the full range of radio fluxes, the rQSOs have a higher detection fraction than the cQSOs, although the uncertainties are large at the faintest fluxes in the $\mathrm{C} 3 \mathrm{GHz}$ survey.

(see Figure 4 therein), who found a factor $\approx 3$ enhancement in the FIRST radio-detection fraction of rQSOs compared to their cQSO and blue QSO samples, but with much higher source statistics at the brighter end. We note the apparent detection fraction discontinuity in the overlapping region of fluxes between $\mathrm{C} 3 \mathrm{GHz}$ and $\mathrm{S} 82$ is consistent within the large statistical uncertainties (only $3 \mathrm{cQSOs}$ and 3 rQSOs are enclosed by this overlapping region). However there may be small systematic contributions due to different deconvolution and source extraction methods between the two radio surveys which are especially prominent at the faint end of the S82 sample.

Fig. 6 shows the $1.4 \mathrm{GHz}$ radio luminosity (see Section 2.3.2) versus redshift for the cQSOs and rQSOs. The FIRST flux threshold is shown for reference, illustrating that many sources from both surveys are too faint to be detected by FIRST. To indicate the radio emission that we might expect from powerful star-formation (SF), we plot the $100 \mathrm{M}_{\odot} \mathrm{yr}^{-1}$ star-formation rate (SFR) line, with mainly low redshift $\mathrm{C} 3 \mathrm{GHz}$ sources falling below this line. This analysis suggests that the radio emission from the majority of the radiodetected QSOs is dominated by the AGN, particularly for the S82
QSOs; see Section 4.1 for more detailed constraints on the origin of the radio emission.

In Klindt et al. (2019), the enhancement in the radio-detection fraction arose from systems around the radio-loud/radio-quiet threshold. To quantify how many of the rQSOs and cQSOs in our sample are 'radio-quiet' we adopted the same "radio-loudness" parameter $(\mathcal{R})$ as that used in Klindt et al. (2019), defined as the dimensionless quantity:

$\mathcal{R}=\log _{10} \frac{1.4 \times 10^{16} L_{1.4 \mathrm{GHz}}\left[\mathrm{W} \mathrm{Hz}^{-1}\right]}{L_{6 \mu \mathrm{m}}\left[\mathrm{erg} \mathrm{s}^{-1}\right]}$.

We also used the same radio-loud/radio-quiet threshold of $\mathcal{R}=-4.2$, equivalent to a mechanical-to-radiative power ratio of $P_{\text {mech,sync }} / P_{\text {rad,L6 } 4 \mathrm{~m}} \approx 0.001$, which is broadly consistent with the classical threshold often defined using a $5 \mathrm{GHz}$-to- $2500 \AA$ flux ratio, but is less susceptible to obscuration from dust (see Klindt et al. 2019 for full details). In Fig. 7 we plot $L_{6 \mu \mathrm{m}}$ versus $L_{1.4 \mathrm{GHz}}$ for the $\mathrm{S} 82$ and $\mathrm{C} 3 \mathrm{GHz}$ parent and colour-selected samples and indicate our adopted radio-loud/radio-quiet threshold. From this plot it is clear that the extreme radio-loud population is dominated by the 


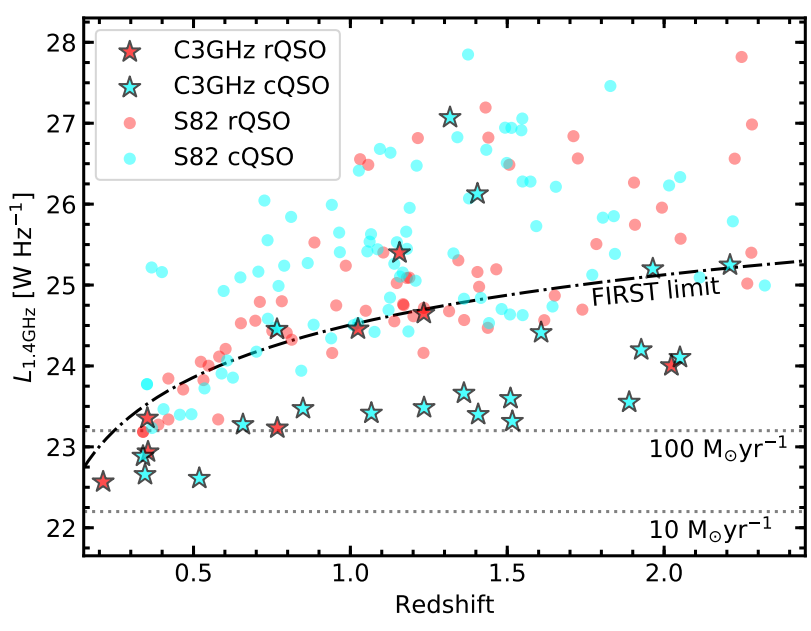

Figure 6. Radio luminosity at $1.4 \mathrm{GHz}$ versus redshift for the $\mathrm{S} 82$ (filled circles) and $\mathrm{C} 3 \mathrm{GHz}$ (stars) QSOs. The $1.4 \mathrm{GHz}$ luminosity for the $\mathrm{C} 3 \mathrm{GHz}$ sample was either taken from the VLA-COSMOS $1.4 \mathrm{GHz}$ catalogue (Schinnerer et al. 2007), or calculated from the $3 \mathrm{GHz}$ flux by assuming a uniform spectral slope of $\alpha=-0.7$. The dotted lines indicate the radio luminosity representing star-formation rates of $10 \mathrm{M}_{\odot} \mathrm{yr}^{-1}$ and $100 \mathrm{M}_{\odot} \mathrm{yr}^{-1}$, converted using the Kennicutt \& Evans (2012) relation. The dot-dashed curve displays the FIRST $5 \sigma$ detection threshold; the majority of the $\mathrm{C} 3 \mathrm{GHz}$ sources and $\sim 25 \%(36 / 143)$ of the S82 sources lie below this curve.

cQSOs (67\%), which could indicate a transition between the red and control QSO populations.

Splitting the $\mathrm{S} 82$ and $\mathrm{C} 3 \mathrm{GHz}$ radio-detected sources into four contiguous $\mathcal{R}$ bins (with boundaries $\mathcal{R}<-5,-5<\mathcal{R}<-4.2$, $-4.2<\mathcal{R}<-3.4$ and $\mathcal{R}>-3.4$ ), we calculated the enhancement in the radio-detection fraction of the full colour-selected sample for the rQSOs. From the lowest bin $(\mathcal{R}<-5)$ to the highest bin $(\mathcal{R}>-3.4)$, we found an enhancement of $2.1_{-0.6}^{+1.0}, 5.8_{-1.3}^{+1.9}, 3.1_{-0.8}^{+1.2}$ and $1.6_{-0.4}^{+0.7}$, respectively which is displayed in Fig. 8. The shaded pink region compares our results to Klindt et al. (2019), who probe down to $\mathcal{R} \sim-4.5$. We find good agreement with the enhancement seen in Klindt et al. (2019) (within $1 \sigma$ uncertainties), despite using an optically fainter QSO sample, but also show that with $\mathrm{C} 3 \mathrm{GHz}$ we can push to the levels where SF is likely to be important (detection limit of $\mathrm{C} 3 \mathrm{GHz}$ at $z=1.5: 3.9 \times 10^{23} \mathrm{WHz}^{-1}$, see Fig. 6). Therefore we confirm that the radio enhancement seen in rQSOs appears to arise around the radio-loud/radio-quiet threshold with tentative evidence for a decrease at both $\mathcal{R}<-5$ and $\mathcal{R}>-3.4$; the latter confirmed with better source statistics in Klindt et al. (2019). This is also confirmed with a larger sample using deep LOFAR data, which gives qualitatively the same result, demonstrating there is a decrease in the radio enhancement of rQSOs for $\mathcal{R}<-5$ (Rosario et al. 2020).

\subsection{Median radio stacking}

The $\mathrm{C} 3 \mathrm{GHz}$ data probes radio flux values for individually detected sources down to $\mu \mathrm{Jy}$ levels, allowing for the majority of the QSOs to be detected in the radio band. However, $\mathrm{C} 3 \mathrm{GHz}$ only covers a small area with modest source statistics, whereas S82 covers a $\sim 35$ times larger area, resulting in a higher number of sources, albeit 12 times shallower. Radio stacking allows us to compare the average properties of undetected S82 QSOs that would have been detected at the $\mathrm{C} 3 \mathrm{GHz}$ depth.

We stacked the S82 data of the radio-undetected QSOs fol-
Table 3. The number of S82 rQSOs and cQSOs in each of the morphology classes, with the overall fraction of the colour-selected sample shown. The fractional difference between the rQSOs and the CQSOs is $4.2_{-0.9}^{+1.3}$ for the faint category, $2.9_{-0.6}^{+0.9}$ for the compact category and $2.2_{-0.8}^{+1.6}$ for the extended category.

\begin{tabular}{l|ll|ll}
\hline \hline \multirow{2}{*}{ Classification } & \multicolumn{2}{|c|}{ rQSO } & \multicolumn{2}{c}{ cQSO } \\
& Num. & $\%$ & Num. & $\%$ \\
\hline Faint & 28 & $7.5_{-1.2}^{+1.6}$ & 30 & $1.8_{-0.3}^{+0.4}$ \\
Compact & 24 & $6.5_{-1.1}^{+1.5}$ & 34 & $2.0_{-0.3}^{+0.4}$ \\
Extended & 6 & $1.6_{-0.4}^{+0.9}$ & 12 & $0.7_{-0.2}^{+0.3}$ \\
FRII-like & 3 & $0.8_{-0.2}^{+0.8}$ & 6 & $0.4_{-0.1}^{+0.2}$ \\
\hline \hline
\end{tabular}

lowing the procedure defined in Section 2.5. We found median $1.4 \mathrm{GHz}$ flux values of $35.0 \pm 2.7 \mu \mathrm{Jy}(\mathrm{S} / \mathrm{N} \sim 13)$ and $26.1 \pm 1.8 \mu \mathrm{Jy}$ $(\mathrm{S} / \mathrm{N} \sim 15)$ for the $\mathrm{S} 82 \mathrm{rQSOs}$ and cQSOs, respectively. Again, fluxes on the computed stacks are not corrected for any CLEAN or snapshot bias, but we can make a qualitative comparison between the stacks. The final stacked images for the $\mathrm{S} 82$ radio-undetected colour-selected QSOs are shown in Fig. 9, displaying the significant detections. Although the difference is small, the rQSOs appear to be relatively brighter in the radio band than the cQSOs for the undetected population. It is important to note that these radio flux values may be a combination of AGN activity and SF, especially for the $\mathrm{C} 3 \mathrm{GHz}$ stack (see Section 4.1).

We also analysed the redshift dependence of our radioundetected stacks. Splitting the S82 radio-undetected cQSOs and rQSOs into four contiguous redshift bins with boundaries 0.2, 0.5, $0.8,1.5$ and 2.4 , we calculated the $1.4 \mathrm{GHz}$ flux at the median redshift of all QSOs in each bin. For all redshift bins, the undetected rQSOs have a slightly higher relative flux compared to the cQSOs.

\subsection{Radio morphology fractions of red and control QSOs}

Using the FIRST radio data, Klindt et al. (2019) found that the radiodetection enhancement of their rQSOs was due to a higher incidence of compact and faint radio counterparts. On the basis of the FIRST data, sources were classified as compact if their radio emission is unresolved on scales less than the $5^{\prime \prime}$ beam size (i.e. corresponding to $43 \mathrm{kpc}$ at $z=1.5$ ). Using the higher spatial resolution radio data in this study, we can probe down to host-galaxy scales (S82: $16 \mathrm{kpc}$ at $z=1.5$; C3GHz: $7 \mathrm{kpc}$ at $z=1.5$ ), resolving finer-scale structure in many of the FIRST compact sources and testing whether rQSOs still show an enhancement in compactness on these smaller scales. The greater depth of our radio data also allows us to determine the morphology of many systems too faint to detect or categorise with FIRST.

We classified the radio morphologies of the rQSOs and cQSOs following Section 2.4, using the high-resolution $\mathrm{S} 82$ and $\mathrm{C} 3 \mathrm{GHz}$ radio data. The number of objects classified in our four morphology categories is shown in Table 3, with the fractions calculated from the colour-selected QSOs within Stripe 82. Fig. 10 shows the S82 radio morphology fractions: the rQSOs show a clear enhancement in the faint and compact morphology groups by a factor of $4.2_{-0.9}^{+1.3}$ and $2.9_{-0.6}^{+0.9}$, respectively, when compared to the cQSOs, quantitatively consistent with the result from Klindt et al. (2019). However, we now also see a tentative difference in the extended group, with rQSOs showing a factor $2.2_{-0.8}^{+1.6}$ enhancement compared to the cQSOs. This enhancement is not seen at the lower resolution of FIRST, where Klindt et al. (2019) found the two samples to be consistent in the extended category (defined as radio 


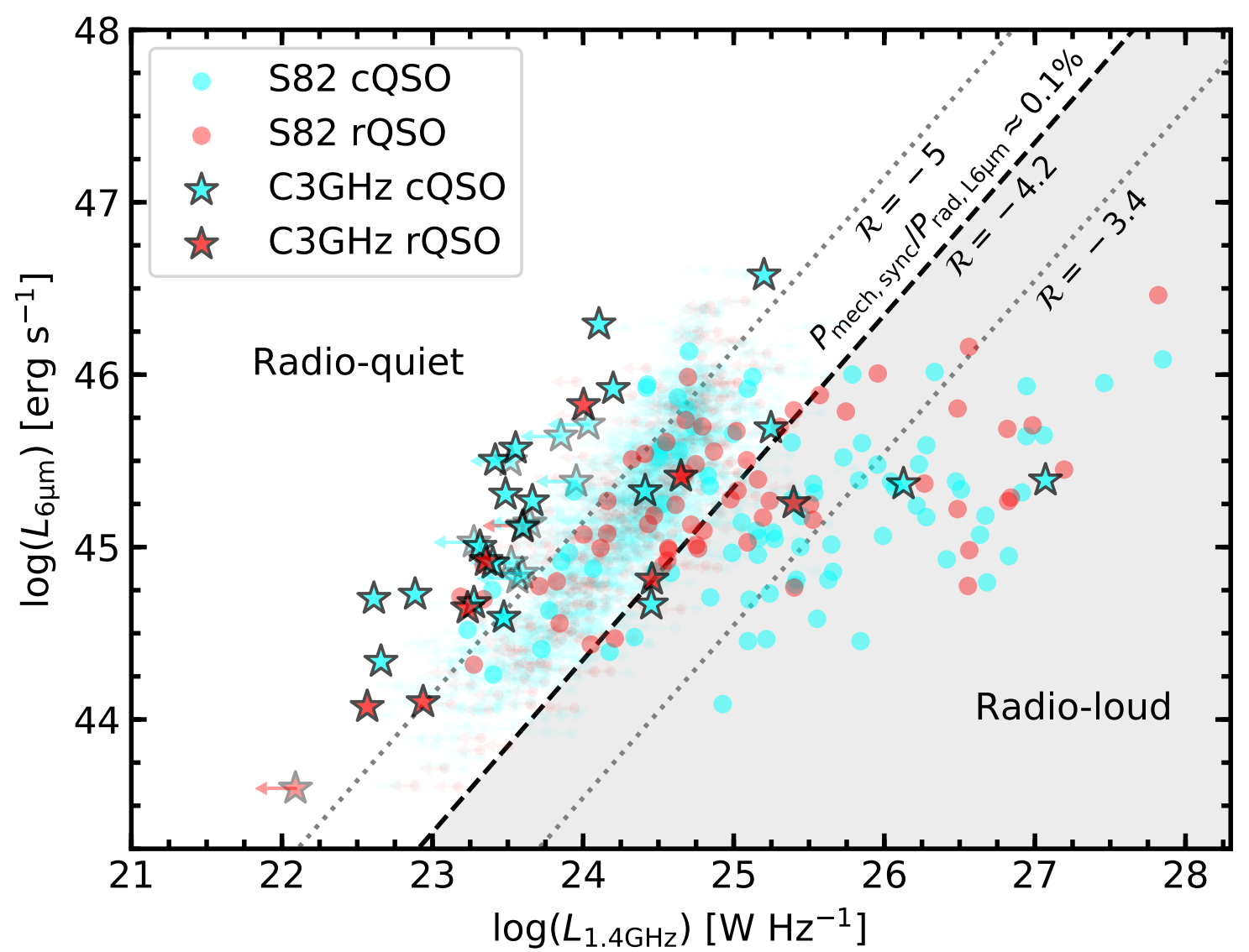

Figure 7. $L_{6 u \mathrm{~m}}$ versus $L_{1.4 \mathrm{GHz}}$ for the $\mathrm{S} 82$ (filled circles) and $\mathrm{C} 3 \mathrm{GHz}$ (stars) sources. The faded points with arrows indicate the properties of the radio-undetected sources, with upper limits derived from the RMS mosaics. The dashed bold line indicates the radio-loud/radio-quiet threshold, defined as a mechanical-toradiative power of $0.1 \%$ ( $\mathcal{R}=-4.2$; see Section 3.3 in Klindt et al. 2019 for details). Additional dotted lines at $\mathcal{R}=-5$ and $\mathcal{R}=-3.4$ represent the boundaries of the bins used in our analysis, between which we find the highest enhancement, as demonstrated in Fig. 8.

emission on scales larger than $\left.5^{\prime \prime}\right)$. Although this enhancement is around the $2 \sigma$ significance level, this may indicate fundamental differences between rQSOs and cQSOs on 1 .' $^{\prime}-5^{\prime \prime}$ scales (16-40 kpc at $z=1.5$ ) that can be explored with even higher spatial resolution. There is no difference in the FRII-like category within the $1 \sigma$ error bars, consistent with that found in Klindt et al. (2019).

As shown in Fig. 8, we found the biggest enhancement in the radio-detection fraction for rQSOs arises around the radioloud/radio-quiet threshold $(-5<\mathcal{R}<-3.4)$. Looking at the morphologies of sources in these bins, we find that the radio enhancement is entirely driven by compact and faint sources, in agreement with Klindt et al. (2019) but pushing to even lower $\mathcal{R}$ values.

Fig. 11 shows cutouts of the S82 QSOs with extended radio morphologies, all of which are radio-loud and detected by FIRST. The FIRST classification is shown in the bottom right corner of each cutout, indicating which sources are reclassified in the S82 data. Many of the S82 extended sources were FIRST compact sources where we can now resolve more diffuse emission. On the basis of the FIRST morphology classifications, none of the rQSOs classified as extended in the S82 data were found to show extension beyond the 5 " resolution of the FIRST data. This is compared to $75 \%(9 / 12)$ of the cQSOs, indicating that the radio emission of the extended rQSOs is on smaller scales than the cQSOs ( $\left.\approx 11^{\prime \prime} 8-5^{\prime \prime} ; 16-43 \mathrm{kpc}\right)$; indeed, although limited by small source statistics, our data suggests a factor $\approx 10$ times more rQSOs are extended on these scales than cQSOs.
We also investigated the radio morphology fractions using the $\mathrm{C} 3 \mathrm{GHz}$ data: the rQSOs still show an enhancement in the compact category of $2.5_{-0.9}^{+1.0}$ compared to the cQSOs (7/8 compact rQSOs and $8 / 20$ compact cQSOs), but now going down to radio fluxes $\approx 12$ times fainter and a spatial resolution $\approx 2.4$ times higher than that of S82. There are no significant differences in the faint and extended groups, however there are too few sources to draw any statistically significant conclusions.

\section{DISCUSSION}

We have used high-resolution, deep radio data to explore differences between red QSOs and typical QSOs detected in the SDSS DR14 quasar survey. Using the VLA Stripe 82 and VLA-COSMOS $3 \mathrm{GHz}$ data, we have explored the radio properties of QSOs to a depth of $\sim 3$ and 30 times that of FIRST, down to scales of $16 \mathrm{kpc}$ and $7 \mathrm{kpc}$ at $z=1.5$, respectively. With these data we confirm the results from Klindt et al. (2019), but down to lower radio luminosities and hence unexplored regions of the radio-loudness plane ( $L_{6 \mu \mathrm{m}}$ vs $\left.L_{1.4 \mathrm{GHz}}\right)$. From this we can gain new insight into the origin and scale of the radio emission in radio-quiet systems.

We find a significant enhancement in the radio emission of red QSOs down to low radio fluxes ( $\sim 0.3 \mathrm{mJy}$ in S82). Looking at the radio-detection fraction as a function of flux for FIRST, S82 and 


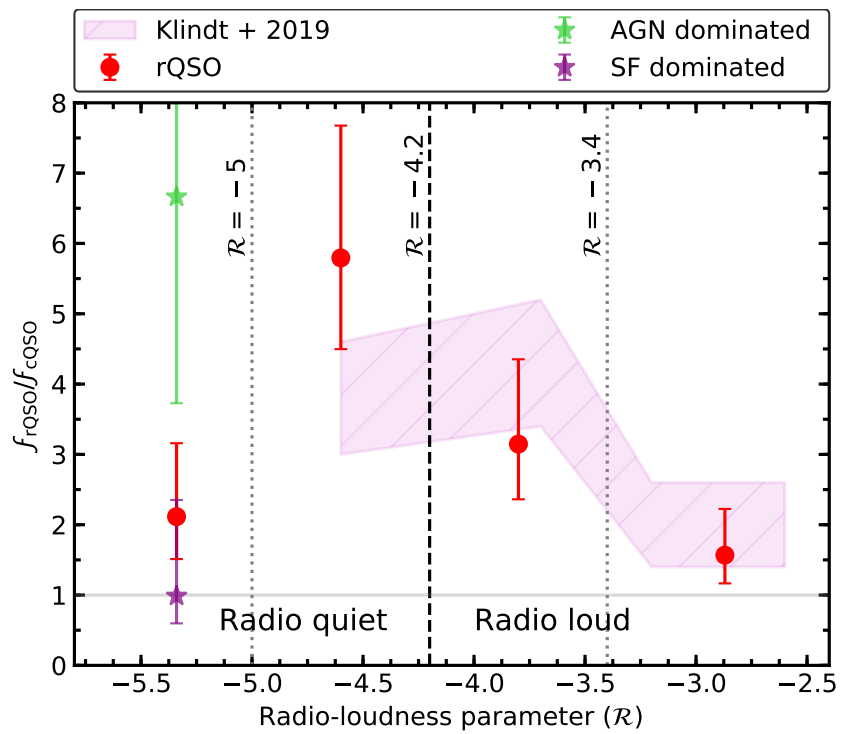

Figure 8. The radio-detection enhancement for rQSOs as a function of the radio-loudness parameter, $\mathcal{R}$ (see Section 3.1). The red circles give the overall enhancement, which peaks around the radio-loud/radio-quiet threshold, and decreases in both the highest and lowest bin, a trend also seen using deep LOFAR data in our companion study by Rosario et al. (2020). The shaded pink region represents the result from Klindt et al. (2019), which is consistent with our data for the overlapping regions of $\mathcal{R}$, within the $1 \sigma$ uncertainties. Therefore we confirm the result at the brighter end, but also push much deeper with the $\mathrm{C} 3 \mathrm{GHz}$ data. In the lowest bin $(\mathcal{R}<-5)$, the $\mathrm{C} 3 \mathrm{GHz}$ sources that are SF dominated are indicated by the purple star, and the sources predominately AGN dominated are indicated by the green star (see Section 4.1 for details). The vast majority of the SF-dominated sources lie at $\mathcal{R}<-5$ suggesting that the radio-detection enhancement seen at $\mathcal{R} \approx-5$ to -3.4 is due to AGN processes. We argue in Section 4.1 that the decrease in the radio enhancement seen at $\mathcal{R}<-5$ is therefore not intrinsic but is due to SF diluting the overall radio emission.
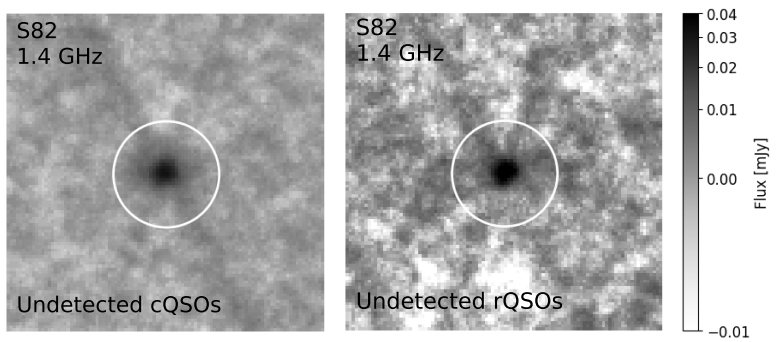

Figure 9. The resulting $0.5 \times 0.5$ images of the median radio $(1.4 \mathrm{GHz})$ stacked radio-undetected cQSOs (left) and radio-undetected rQSOs (right) in the S82 field. The white circle has a 10" radius and the colour bar has an arcsinh scaling. A two-dimensional Gaussian was fitted to the images giving a peak $1.4 \mathrm{GHz}$ flux density of $35.0 \pm 2.7 \mu \mathrm{Jy}$ and $26.1 \pm 1.8 \mu \mathrm{Jy}$ for the $\mathrm{S} 82$ rQSOs and cQSOs, respectively, although this has not been corrected for any CLEAN or snapshot bias (see White et al. 2007).

$\mathrm{C} 3 \mathrm{GHz}$, we find an enhancement that is broadly constant at a factor of $\approx 3$, although the uncertainties are large at faint radio fluxes in the $\mathrm{C} 3 \mathrm{GHz}$ field (see Fig. 5). We pushed this limit further by stacking the radio-undetected S82 rQSO and cQSO sources, which resulted in a higher average relative flux for the rQSOs. We also explored the radio-detection enhancement in the rQSOs as a function of the radio-loudness parameter $\mathcal{R}$, where we confirm the enhancement peaks around the radio-quiet threshold $-5<\mathcal{R}<-3.4$ (enhancement of $\sim 3-6)$; however, for our lowest bin $(\mathcal{R}<-5)$, which probes a magnitude below that explored in Klindt et al. (2019), we see a decrease in the enhancement by a factor of $\sim 3$. This suggests that the fundamental differences between rQSOs and cQSOs become apparent at $\mathcal{R}<-3.4$, possibly due to a different process starting to dominate the radio emission. However, whether this process is due to AGN-driven winds, frustrated jets, star-formation or a coronal component (to name a few) is unclear (Zakamska \& Greene 2014; Kellermann et al. 2016; Laor et al. 2019; Panessa et al. 2019; Jarvis et al. 2019).

An enhanced fraction of broad absorption line QSOs (BALQSOs; known to host powerful winds) have been found in red QSOs (Urrutia et al. 2009), which could be evidence that red QSOs are more wind dominated than typical QSOs. Although BALQSOs are virtually all radio-quiet, they have also been found to show a remarkably similar enhancement in the radio as that found for red QSOs (Klindt et al. 2019; Morabito et al. 2019). Further corroborating but independent evidence for wind-dominated sources becoming more relevant at lower $\mathcal{R}$ values comes from Mehdipour \& Costantini (2019), who showed a significant inverse correlation between the $\mathrm{X}$-ray measured column density of the ionised wind in AGN and the radio-loudness parameter. On the basis of this we may therefore expect a larger fraction of the rQSOs to have more extreme winds than typical QSOs, which would be consistent with rQSOs representing a younger phase in the QSO evolutionary scenario.

To try and constrain the nature of the differences in the radio emission we investigated the radio morphologies. Probing down to arcsecond scales and sensitivity limits at least a factor $\approx 3$ deeper than FIRST has allowed us to undertake a thorough test of the morphology result from Klindt et al. (2019), who found that rQSOs showed an enhancement in the compact and faint radio morphology classes when compared to typical QSOs; we find that 40\% (24/61) of our rQSOs are compact on scales of $\sim 16 \mathrm{kpc}$ at $z=1.5$, the resolution of our data. We found good agreement with their result, but we also found a tentative enhancement (at the $2 \sigma$ statistical level) in the extended morphologies of rQSOs which is not seen at the resolution of FIRST, suggesting that we are starting to resolve the scales of the radio structures that are driving these differences $(16-43 \mathrm{kpc}$ at $z=1.5)$.

We found that none of the extended rQSOs are classified as extended at the resolution of FIRST, which again indicates that the radio emission in rQSOs occurs on scales $16-43 \mathrm{kpc}$ (at $z=1.5$ ). This enhancement in the extended rQSOs is driven by the improved resolution of the survey, with sources that may have been classified as compact at the resolution of FIRST now reclassified as extended (see Appendix C). The compactness of the radio emission could imply that these objects are in a young phase compared to typical bluer QSOs, which are more likely to have extended radio jets, consistent with an evolutionary scenario. Using data from Siemiginowska et al. (2016), who investigate young radio sources with known kinematic radio jet ages, a crude linear relationship between jet age and the size of the radio emission can be constructed. Applying this relation to the scales on which these radio differences occur $(16-43 \mathrm{kpc}$ at $z=1.5$ ) yields a range of $0.2-0.5 \mathrm{Myrs}$ as a rough estimate for the age of the extended radio emission in the rQSOs. We note that no correction for orientation has been made, and so this represents a lower limit; however, this timescale is broadly consistent with other work that estimate a rQSO phase duration of a few Myrs (Hopkins et al. 2006; Glikman et al. 2012).

Overall our results add weight to the emerging picture that 

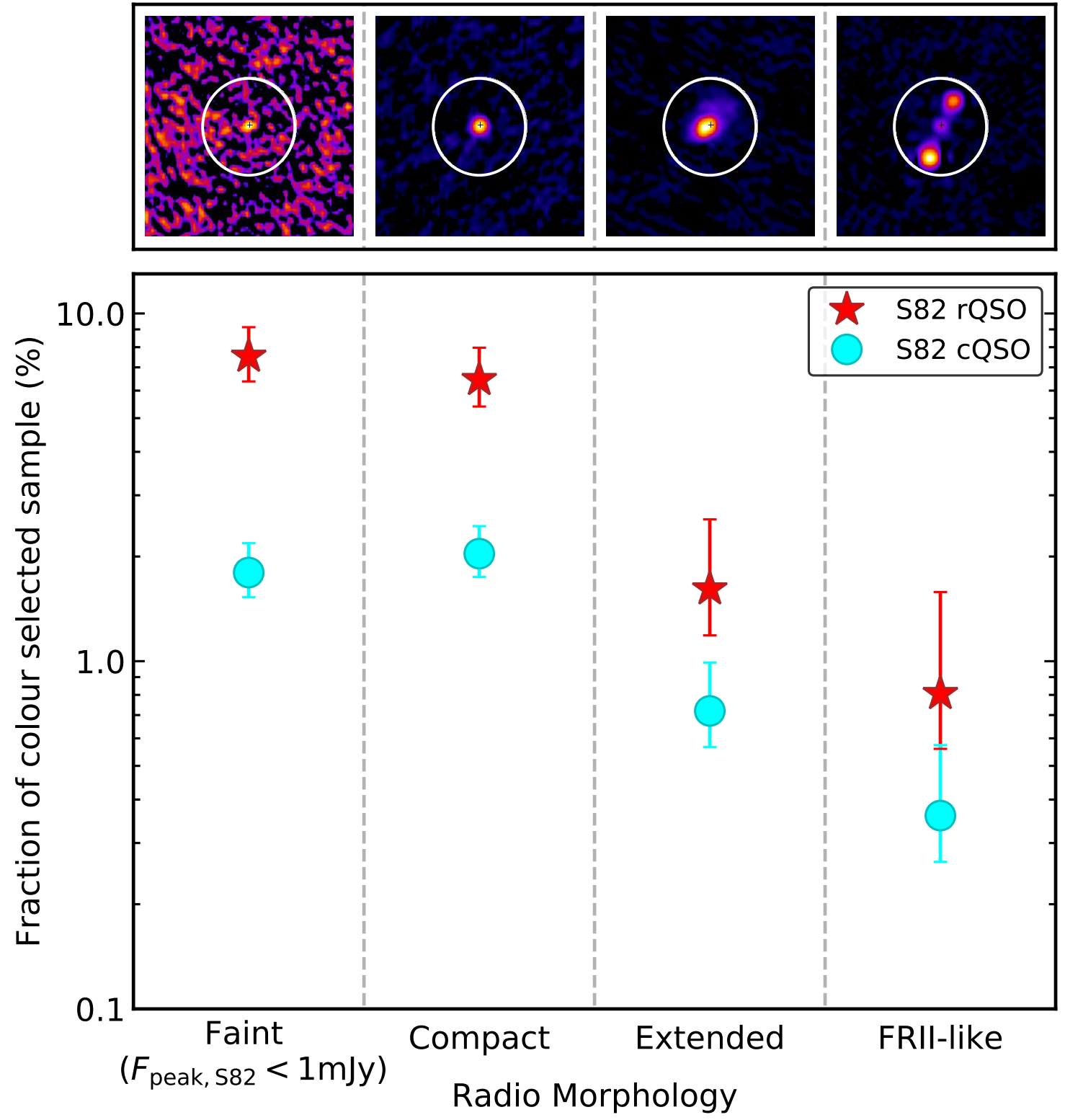

Figure 10. Radio morphology fractions for the S82 rQSOs and cQSOs. The thumbnails display $45^{\prime \prime} \times 45^{\prime \prime}$ examples of the four morphology types for the S82 VLA data (Hodge et al. 2011). The white circles represent a 10" radius. The error bars were calculated using the method described in Cameron (2011) and correspond to the equivalent $1 \sigma$ uncertainties on the ratio. The rQSOs show a strong factor $\approx 3$ enhancement in the faint and compact classes and a less significant factor $\approx 2$ enhancement in extended radio morphologies; however, there are no differences between the rQSOs and cQSOs in the FRII-like group within the $1 \sigma$ error bars although we note that there are only 9 FRII-like QSOs in total; however, we note that we also did not see any significant difference in the FRII-like category between rQSOs and cQSOs in our companion study (Klindt et al. 2019) which had significantly improved source statistics.

red QSOs are fundamentally different to typical QSOs and cannot be explained by the simple orientation model alone. These differences in the radio properties are most likely to be driven by differences in the accretion or "environment" (see Footnote 1); since Klindt et al. (2019) demonstrated that differences in the accretion appear unlikely, environmental differences are the most probable cause. Below, we focus our discussion on the origin of the radio emission in these systems (Section 4.1) and constrain the SF contribution for the $\mathrm{C} 3 \mathrm{GHz}$ sample (Section 4.2). To maximise the source statistics we use the $\mathrm{S} 82$ and $\mathrm{C} 3 \mathrm{GHz}$ parent samples rather than focus on the colour-selected QSOs (see Table 1); however, we do explore whether SF can explain the rQSO enhancement ${ }^{5}$.

\subsection{SF contribution to the radio emission in radio-quiet QSOs}

Using the deeper data, we find that the radio enhancement seen in rQSOs is stronger for sources around the radio-loud/radio-quiet

\footnotetext{
5 We note that our results in Sections 4.1 and 4.2 also hold when restricting the analysis to the colour-selected samples, but with poorer source statistics.
} 
Extended rQSOs
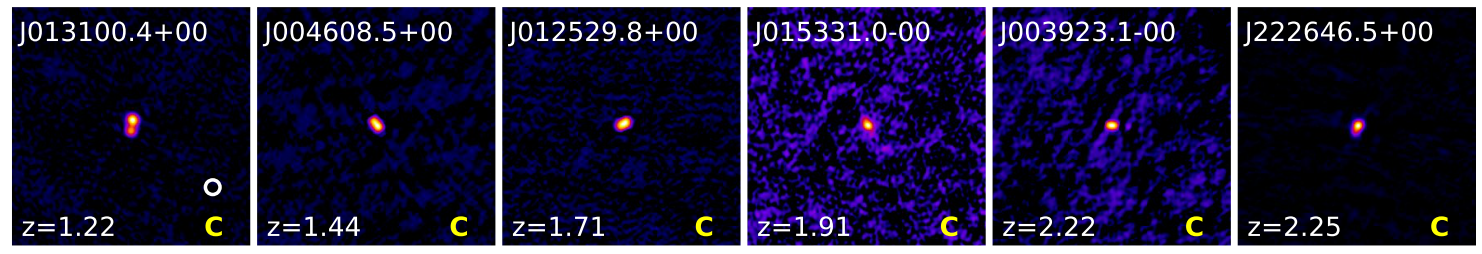

\section{Extended cQSOs}
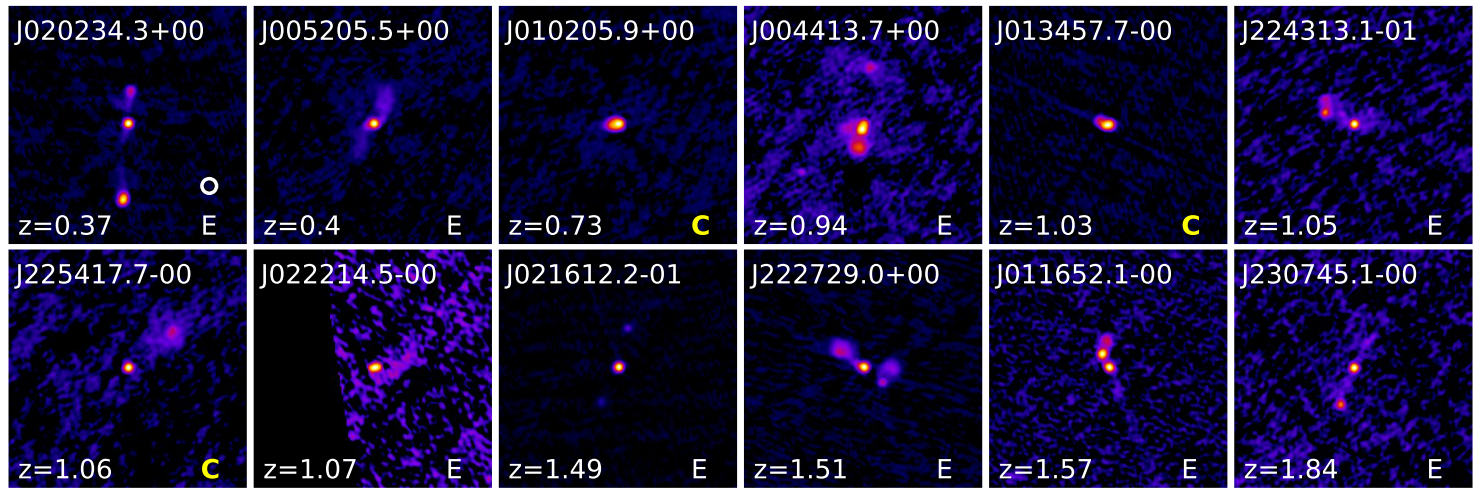

Figure 11. Thumbnails $\left(1^{\prime} \times 1^{\prime}\right)$ of S82 extended rQSOs (top) and cQSOs (bottom) using the S82 data (Hodge et al. 2011). The white circle in the first cutout represents the 1 ". 8 beam size. The redshift is shown in the bottom left corner and the VLA name is shown in the top left corner. The classification from visually inspecting the FIRST data (Becker et al. 1995) is shown in the bottom right corner of the image; C (compact) or E (extended). The sources that were reclassified from FIRST with the higher resolution data are highlighted in yellow, showing that $0 / 6$ of the rQSOs were classified as extended in the FIRST data, compared to $9 / 12$ of the cQSOs which indicates that the rQSOs are only extended on $\approx 1$ ". $8-5^{\prime \prime}$ scales.

threshold $(-5<\mathcal{R}<-3.4)$, peaking in the bin just below the threshold and dropping at lower and higher values of $\mathcal{R}$ (see Section 3.1). For $\mathcal{R}>-4.2$, all $\mathrm{C} 3 \mathrm{GHz}$ and $\mathrm{S} 82$ parent sample QSOs have $L_{1.4 \mathrm{GHz}} \gtrsim 2 \times 10^{24} \mathrm{WHz}^{-1}$, which corresponds to an equivalent SFR of $\gtrsim 1000 \mathrm{M}_{\odot} \mathrm{yr}^{-1}$, suggesting that the vast majority are likely to be AGN dominated. However at $\mathcal{R}<-4.2$, it is unclear whether $\mathrm{SF}$ or AGN processes are the origin of the radio enhancement. We can make progress in understanding the origin of the radio enhancement by utilising the Herschel far-IR (FIR) data in COSMOS and Stripe 82 and constrain the contribution from SF.

In this analysis we utilised rest-frame $(8-1000 \mu \mathrm{m})$ IR luminosity constraints on SF from Stanley et al. (2015) (covering 53/70 of our C3GHz parent QSO sample), and Smolčić et al. (2017b) (covering $47 / 70$ of our $\mathrm{C} 3 \mathrm{GHz}$ parent QSO sample), who both fit an AGN and SF component to the SEDs of X-ray and radio AGN, respectively. The IR luminosities were taken preferentially from Stanley et al. (2015), resulting in a total of $61 / 70$ of our C3GHz parent sample with IR data from one of the two catalogues. Since S82 is much shallower than $\mathrm{C} 3 \mathrm{GHz}$ and does not have deep Herschel data, we only use this sample to probe the brighter IR end of the QSO population.

The tight radio-FIR relationship for star-forming galaxies provides a way to identify SF-dominated QSOs. In Fig. 12, we plot the SF luminosity constraints of $61 \mathrm{C} 3 \mathrm{GHz}$ parent QSOs with IR data. We also plot on the line indicating a 1:1 relation between the 1.4 $\mathrm{GHz}$ radio emission expected from the SFR of a source, which was calculated by converting the AGN host component of the IR luminosity to a radio luminosity using the relation given in Delhaize et al. (2017), assuming $q_{\mathrm{TIR}}=2.64$ (Bell 2003). This relation was calibrated on the VLA-COSMOS $3 \mathrm{GHz}$ data and so is suitable for

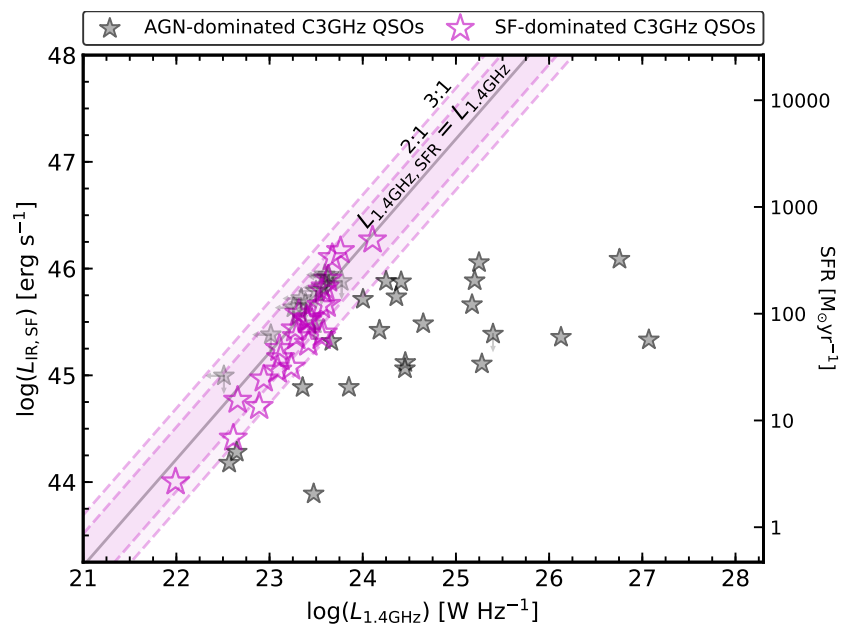

Figure 12. IR luminosity from star-formation versus $1.4 \mathrm{GHz}$ luminosity for the $\mathrm{C} 3 \mathrm{GHz}$ parent QSOs. Only QSOs with Herschel coverage are shown (61/70 C3GHz parent sample QSOs). The radio luminosity upper limits were calculated from the $3 \mathrm{GHz}$ RMS mosaics, and are converted to $1.4 \mathrm{GHz}$ assuming a uniform spectral index of -0.7 . The grey solid line indicates a 1:1 relation between the measured $1.4 \mathrm{GHz}$ radio emission and that expected from the SFR of a source. The SFR was calculated from the IR luminosity using the Kennicutt (1998) conversion and scaling to a Chabrier IMF. The shaded purple region illustrates a factor of three deviation from this line, the range adopted here for a source classified as SF dominated. Excluding sources with an upper limit on the SFR, 27 QSOs lie within this region, illustrated by empty purple stars, giving an estimate of the fraction of QSOs with radio emission that is potentially SF dominated of $44 \%$. 
our sample. We define the origin of the radio emission as dominated by $\mathrm{SF}$ if the measured radio luminosity is within a factor of 3 of the $1: 1$ relation; resulting in $27 / 61(\approx 44 \%)$ sources classified as SF dominated. If a source has an upper limit on the SFR then the origin of the radio emission is classified as uncertain.

In order to further explore the origin of the radio emission down to low $\mathcal{R}$ values, in Fig. 13 we plot $L_{\mathrm{IR}, \mathrm{SF}} / L_{1.4 \mathrm{GHz}}$ as a function of $\mathcal{R}$ for the $\mathrm{C} 3 \mathrm{GHz}$ parent sample QSOs with IR coverage. Our selection of SF-dominated QSOs (from Fig. 12) is shown as the shaded purple horizontal region and the dashed vertical line indicates the radio-loud/radio-quiet threshold $(\mathcal{R}=-4.2)$. The QSOs with radio emission dominated by SF are indicated by the empty purple stars. As is clear from this figure, all but one of the SFdominated QSOs lie at $\mathcal{R}<-5$, but it is also apparent that not all QSOs that lie below this line will have radio emission dominated by SF. This suggests that SF only significantly contaminates the radio emission in QSOs at $\mathcal{R} \lesssim-5$. Since the $\mathrm{C} 3 \mathrm{GHz}$ data only covers a small area, it will miss the most extreme star-forming QSOs: therefore to test whether this boundary in $L_{6 \mu \mathrm{m}}-L_{1.4 \mathrm{GHz}}$ does indeed isolate the majority of the star-forming population, we take a sample of 148 Herschel-observed QSOs in Stripe 82 (sample from Dong $\& \mathrm{Wu}$ 2016), requiring $W 1, W 2, W 3 \mathrm{SNR}>2$ and $0.2<z<2.4$. We calculated $L_{6 \mu \mathrm{m}}$ for these QSOs following the same approach as Section 2.1.2. After removing the AGN contribution to the FIR luminosity, Dong \& Wu (2016) then convert this to a SFR using the Kennicutt (1998) equation. After converting the SFRs into radio emission using the Kennicutt \& Evans (2012) relation, we find 94\% (139/148) of the sample have $\mathcal{R}<-5$ (full range: $-6.6<\mathcal{R}<-4.8$ ). Since we assume that all of the radio emission comes from SF, this gives an upper limit, demonstrating that the radio emission from QSOs with $\mathcal{R}>-5$ is dominated by AGN processes.

Using this knowledge, we now try to understand the decrease in the radio-detection fraction for the rQSOs at $\mathcal{R}<-5$ (see Section 3.1); here we split the $\mathrm{C} 3 \mathrm{GHz}$ sources at $\mathcal{R}<-5$ into those that are SF dominated and those that are AGN dominated. Fig. 8 displays the radio-detection enhancement in the rQSOs for the different bins considered in Section 3.1. The enhancement in the purely AGN-dominated $\mathrm{C} 3 \mathrm{GHz}$ sources is shown as the green star which, although limited by source statistics, is consistent with the enhancement at $-5<\mathcal{R}<-3.4$. When looking at the purely SF-dominated $\mathrm{C} 3 \mathrm{GHz}$ sources (shown as the purple star), the enhancement becomes consistent with unity, suggesting that differences in the radio properties is not predominantly due to SF. Therefore, as AGN processes clearly dominate the radio emission at higher $\mathcal{R}$ values, the decrease in the enhancement seen at $\mathcal{R} \lesssim-5$ could be due to an increase in the relative contribution to the radio emission from SF as the AGN component becomes comparably weak; i.e. the decrease in the radio enhancement at $\mathcal{R} \lesssim-5$ is not intrinsic but is due to SF diluting the overall radio emission. However, larger QSO samples with faint radio and IR constraints are required to verify this hypothesis.

\subsection{Constraining the SF contribution to the radio emission in the overall QSO population}

With the additional information on the number of SF-dominated QSOs, we can give an estimate on the overall fraction of QSOs where the radio emission is either AGN dominated or SF dominated. Fig. 14 displays the fraction of all the $\mathrm{S} 82$ and $\mathrm{C} 3 \mathrm{GHz}$ parent QSOs within the different radio emission categories: radio-undetected, faint $\left(F_{1.4 \mathrm{GHz}}<1 \mathrm{mJy}\right)$, compact, extended or FRII-like. For the bright $\left(F_{1.4 \mathrm{GHz}}>1 \mathrm{mJy}\right)$ source morphologies we used the $\mathrm{S} 82$ res- olution (1.'8) for all sources. Of the radio-detected QSOs, the faint and compact categories have the highest detection fraction. The zoom in plot utilises the $\mathrm{C} 3 \mathrm{GHz}$ depth to further explore the undetected and faint category at the S82 depth, splitting these sources into uncertain, SF dominated or AGN dominated. The uncertain category is defined by sources with an upper limit on the SFR, the SF-dominated sources have a radio luminosity within a factor of 3 of the radio emission expected from the SFR (see Fig. 14) and the AGN-dominated sources are those not uncertain nor SF dominated. The resulting estimate for the percentage of QSOs that have radio emission dominated by SF is $\approx 44 \%$ (27/61), compared to $\approx 25 \%$ $(15 / 61)$ for AGN-dominated faint sources, $\approx 8 \%(5 / 61)$ for AGNdominated bright sources and $\approx 23 \%$ (14/61) that are still uncertain. Including sources that are classified as uncertain, this gives a range of 44-67\% for QSOs that have radio emission dominated by SF, and $33-56 \%$ for QSOs that have radio emission dominated by AGN processes.

While deeper surveys such as $\mathrm{C} 3 \mathrm{GHz}$ are limited by source statistics, this gives an indication of the capabilities of future sensitive, large-area radio surveys to constrain the origin of the radio emission in QSOs. Upcoming analysis of a sample of 90 colourselected QSOs with FIR continuum measurements from the Atacama Large Millimetre Array (ALMA) will help us to cleanly measure the SF contribution.

\section{CONCLUSIONS}

Using high-resolution and deep radio data of SDSS DR14 QSOs, we extended the results from Klindt et al. (2019) to further explore differences in the radio properties of red QSOs compared to typical QSOs at $0.2<z<2.4$. With the VLA S 82 and $\mathrm{C} 3 \mathrm{GHz}$ radio data explored in our study, we probe down to host-galaxy scales and constrain the star-formation contribution to the radio emission. Our main findings are:

- rQSOs show an enhancement in radio emission around the radio-quiet threshold (see Fig. 5, 8): we confirm that rQSOs have a higher radio-detection fraction compared to cQSOs in $\mathrm{S} 82, \mathrm{C} 3 \mathrm{GHz}$ and FIRST within uncertainties. We find that down to the S82 flux limits, this enhancement is broadly constant at a factor of $\approx 3$ (see Section 3.1). Splitting the $\mathrm{S} 82$ and $\mathrm{C} 3 \mathrm{GHz}$ radio-detected sources into four contiguous $\mathcal{R}$ bins, we find that the enhancement arises from sources within $-5<\mathcal{R}<-3.4$, with no significant enhancement at higher or lower $\mathcal{R}$ values. From median stacking of the undetected S82 rQSOs and cQSOs, we find relatively higher flux values for the rQSOs when compared to cQSOs (factor $\sim 1.3$, see Section 3.2).

- rQSOs show differences in their radio morphologies compared to cQSOs down to host-galaxy scales (see Fig. 10, 11): we find that rQSOs show an enhancement in the compact and faint radio morphologies compared to cQSOs, with a fractional difference of around 2-4. We also find an enhancement in the extended group for rQSOs (1.'8-5"; 16-43 kpc at $z=1.5)$, which is not seen at the FIRST resolution, indicating we are probing scales on which these differences occur. Additionally, none of the extended rQSOs are also extended at the FIRST resolution, compared to $75 \%$ for the cQSOs, which suggests a factor $\approx 10$ times more rQSOs are extended on scales of 1."8-5" than cQSOs (see Section 3.3).

- Overall we find that 33-56\% of the DR14 QSOs have radio emission dominated by AGN processes (see Fig. 12, 14): these ranges are constrained from the $\mathrm{C} 3 \mathrm{GHz}$ data where we define a source as being SF dominated if its radio luminosity lies within a 


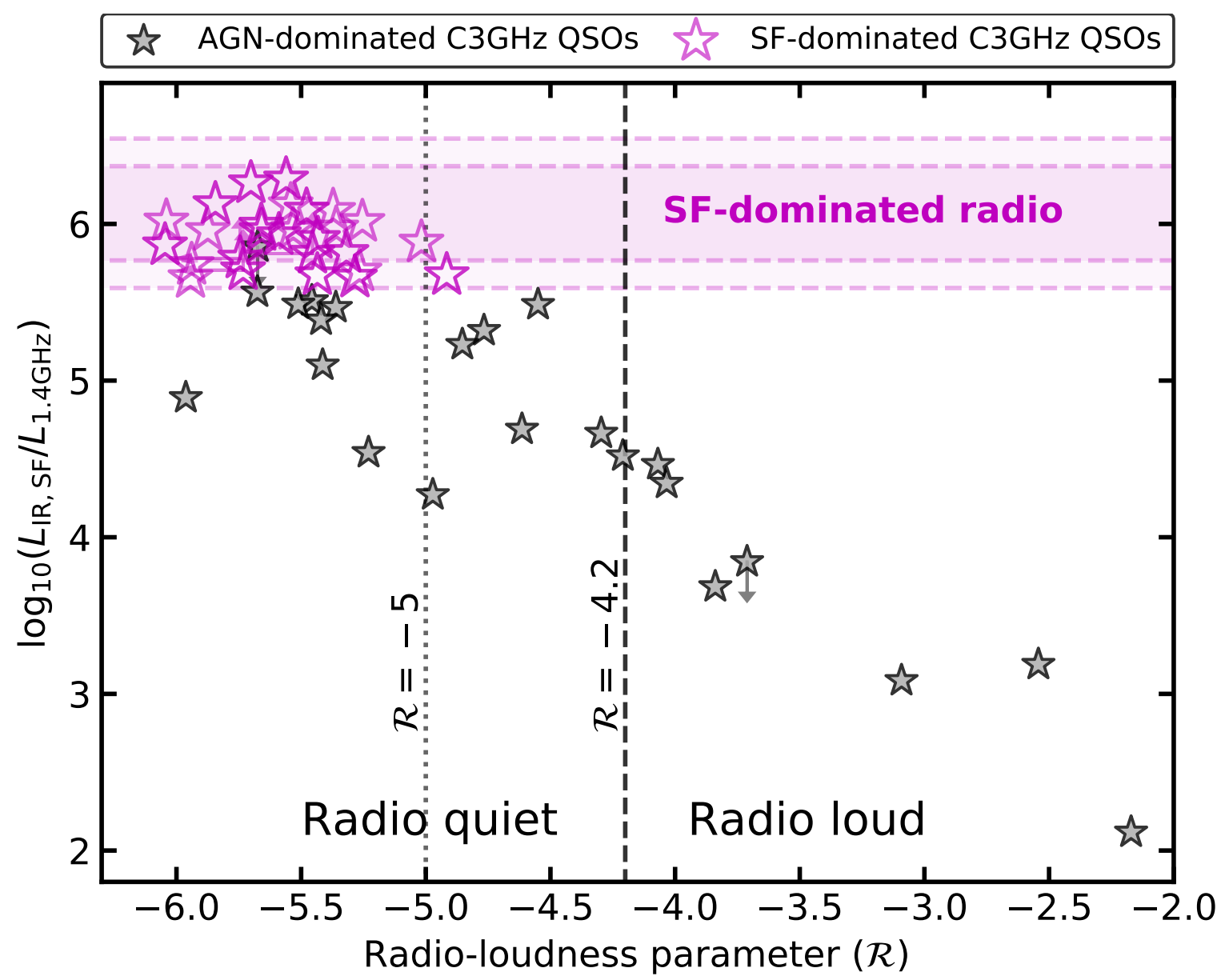

Figure 13. $L_{\mathrm{IR}, \mathrm{SF}} / L_{1.4 \mathrm{GHz}}$ vs $\mathcal{R}$ for the $\mathrm{C} 3 \mathrm{GHz}$ parent sample. Only $\mathrm{C} 3 \mathrm{GHz}$ QSOs with Herschel coverage and a detection in either the IR or radio are shown (50/70 C3GHz parent sample QSOs). We note that all the QSOs with an upper limit on both $L_{\mathrm{IR}, \mathrm{SF}}$ and $L_{1.4 \mathrm{GHz}}$ lie at $\mathcal{R}<-5$. The grey stars represent AGN-dominated C3GHz QSOs and the empty purple stars represent the SF-dominated QSOs. The arrows indicate upper limits on either $L_{\mathrm{IR}, \mathrm{SF}}$ or $L_{1.4 \mathrm{GHz}}$. The purple shaded region displays our selection of a SF-dominated source, as shown in Fig. 12.

factor of three of that expected from SF constrained FIR data and AGN dominated otherwise. This resulted in $44-67 \%$ of $\mathrm{C} 3 \mathrm{GHz}$ parent QSOs classified as having their radio emission dominated by $\mathrm{SF}$, compared to $33-56 \%$ that are AGN dominated. These ranges are due to $\approx 23 \%$ of QSOs still classified as uncertain (upper limit on SFR). We find the QSOs with radio emission dominated by SF emerge about one order of magnitude below the radio-quiet threshold of $\mathcal{R}=-4.2$ (see Fig . 13). Splitting the radio emission from the $\mathrm{C} 3 \mathrm{GHz}$ QSOs into SF dominated and AGN dominated confirms that the decrease in radio enhancement seen at the extremely radioquiet end is likely driven by SF (see Fig. 8). However, future deep radio and rest-frame FIR observations are needed to more directly explore the uncertain category (see Section 4.1).

These fundamental differences in the radio properties of red QSOs predominantly arise in sources with $-5<\mathcal{R}<-3.4$ and are likely driven by differences in the radio-AGN activity between red and typical QSOs. These results provide further evidence for red QSOs representing a young phase in galaxy evolution.

In future work we will use ALMA data to further constrain the star-formation properties of red QSOs and to explore how they differ to typical QSOs. We will also use even higher resolution e-MERLIN radio data to have a more comprehensive understanding of the scale of radio emission in red QSOs down to a few kpc scales, in addition to high-frequency JVLA and 5-band low-frequency GMRT data to search for differences in the radio spectral properties and SEDs of red QSOs.

\section{ACKNOWLEDGEMENTS}

We thank the referee, Eliat Glikman, for her positive and constructive comments. We acknowledge a quota studentship funded by the Science and Technology Facility Council (VAF), the Faculty of Science Durham Doctoral Scholarship (LK), the Science and Technology Facilities Council (DMA, DJR, through grant codes ST/P000541/1 and ST/T000244/1).

Funding for the Sloan Digital Sky Survey has been provided by the Alfred P. Sloan Foundation, the U.S. Department of Energy Office of Science, and the Participating Institutions. SDSS-IV acknowledges support and resources from the Center for HighPerformance Computing at the University of Utah. The SDSS web site is www.sdss.org. SDSS-IV, the primary data set used in this analysis, is managed by the Astrophysical Research Consortium for the Participating Institutions of the SDSS Collaboration including the Brazilian Participation Group, the Carnegie Institution for Science, Carnegie Mellon University, the Chilean Participation Group, the French Participation Group, Harvard-Smithsonian Center for 


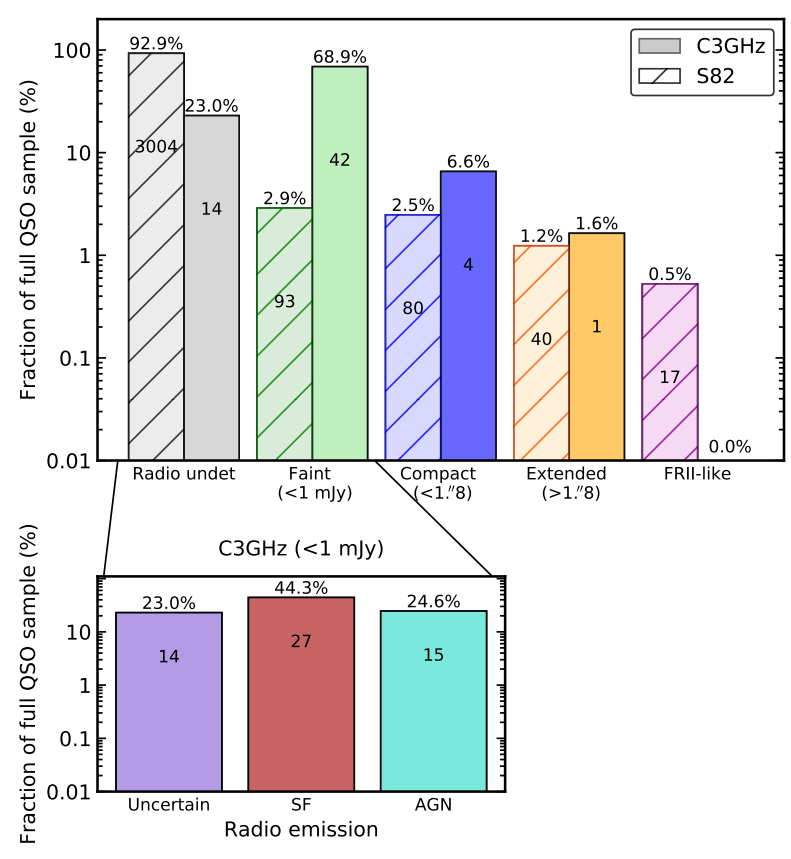

Figure 14. Origin of the radio emission as a fraction of all of the QSOs in the COSMOS and Stripe 82 regions. The radio faint class contains sources that have $F_{1.4 \mathrm{GHz}}<1 \mathrm{mJy}$ and so would be classified as faint in $\mathrm{S} 82$ but can be further explored using the greater depth of $\mathrm{C} 3 \mathrm{GHz}$ (see zoom-in plot). The radio bright $\left(F_{1.4 \mathrm{GHz}}>1 \mathrm{mJy}\right)$ sources are further categorised by radio morphology (see Section 3.3); the majority of the radio bright QSOs have a compact morphology $\left(<1^{\prime \prime} 8\right)$. The number of sources in each category and associated percentage of the full QSO sample are displayed on the bars; we caution that the $\mathrm{C} 3 \mathrm{GHz}$ compact and extended categories are highly uncertain due to poor source statistics. The zoom-in plot utilises the $\mathrm{C} 3 \mathrm{GHz}$ depth to explore the radio-undetected and faint categories, splitting them into uncertain (upper limit on SFR within the SF-dominated region), SF (radio-detected and SF dominated; see Fig. 12) and AGN (radio-detected but not SF dominated).

Astrophysics, Instituto de Astrofísica de Canarias, The Johns Hopkins University, Kavli Institute for the Physics and Mathematics of the Universe (IPMU) / University of Tokyo, the Korean Participation Group, Lawrence Berkeley National Laboratory, Leibniz Institut für Astrophysik Potsdam (AIP), Max-Planck-Institut für Astronomie (MPIA Heidelberg), Max-Planck-Institut für Astrophysik (MPA Garching), Max-Planck-Institut für Extraterrestrische Physik (MPE), National Astronomical Observatories of China, New Mexico State University, New York University, University of Notre Dame, Observatário Nacional / MCTI, The Ohio State University, Pennsylvania State University, Shanghai Astronomical Observatory, United Kingdom Participation Group, Universidad Nacional Autónoma de México, University of Arizona, University of Colorado Boulder, University of Oxford, University of Portsmouth, University of Utah, University of Virginia, University of Washington, University of Wisconsin, Vanderbilt University, and Yale University.

This publication makes use of data products from the Widefield Infrared Survey Explorer, which is a joint project of the University of California, Los Angeles, and the Jet Propulsion Laboratory/California Institute of Technology, funded by the National Aeronautics and Space Administration.
The National Radio Astronomy Observatory is a facility of the National Science Foundation operated under cooperative agreement by Associated Universities, Inc.

\section{REFERENCES}

Alexander D. M., Hickox R. C., 2012, New Astron. Rev., 56, 93

Alexander D. M., et al., 2003, AJ, 125, 383

Assef R. J., et al., 2013, ApJ, 772, 26

Banerji M., McMahon R. G., Hewett P. C., Alaghband-Zadeh S., GonzalezSolares E., Venemans B. P., Hawthorn M. J., 2012, MNRAS, 427, 2275

Banerji M., Alaghband-Zadeh S., Hewett P. C., McMahon R. G., 2015, MNRAS, 447, 3368

Becker R. H., White R. L., Helfand D. J., 1995, ApJ, 450, 559

Bell E. F., 2003, ApJ, 586, 794

Cameron E., 2011, Publ. Astron. Soc. Australia, 28, 128

Dawson K. S., et al., 2013, AJ, 145, 10

Dawson K. S., et al., 2016, AJ, 151, 44

Delhaize J., et al., 2017, A\&A, 602, A4

Dong X. Y., Wu X.-B., 2016, ApJ, 824, 70

Eisenstein D. J., et al., 2011, AJ, 142, 72

Fanaroff B. L., Riley J. M., 1974, MNRAS, 167, 31P

Fanti R., Fanti C., Schilizzi R. T., Spencer R. E., Nan Rendong Parma P., van Breugel W. J. M., Venturi T., 1990, A\&A, 231, 333

Fanti C., Fanti R., Dallacasa D., Schilizzi R. T., Spencer R. E., Stanghellini C., 1995, A\&A, 302, 317

Georgakakis A., Clements D. L., Bendo G., Rowan-Robinson M., Nandra K., Brotherton M. S., 2009, MNRAS, 394, 533

Glikman E., Helfand D. J., White R. L., Becker R. H., Gregg M. D., Lacy M., 2007, ApJ, 667, 673

Glikman E., et al., 2012, ApJ, 757, 51

Glikman E., Simmons B., Mailly M., Schawinski K., Urry C. M., Lacy M., 2015, ApJ, 806, 218

Heywood I., et al., 2016, MNRAS, 460, 4433

Hickox R. C., Alexander D. M., 2018, ARA\&A, 56, 625

Hodge J. A., Becker R. H., White R. L., de Vries W. H., 2008, AJ, 136, 1097

Hodge J. A., Zeimann G. R., Becker R. H., White R. L., 2009, AJ, 138, 900

Hodge J. A., Becker R. H., White R. L., Richards G. T., Zeimann G. R., 2011, AJ, 142, 3

Hopkins P. F., Hernquist L., Cox T. J., Di Matteo T., Robertson B., Springel V., 2006, ApJS, 163, 1

Hopkins P. F., Hernquist L., Cox T. J., Kereš D., 2008, ApJS, 175, 356

Jarvis M. E., et al., 2019, MNRAS, 485, 2710

Jiang L., et al., 2014, ApJS, 213, 12

Kellermann K. I., Condon J. J., Kimball A. E., Perley R. A., Ivezić Ž., 2016, ApJ, 831, 168

Kennicutt Jr. R. C., 1998, ARA\&A, 36, 189

Kennicutt R. C., Evans N. J., 2012, ARA\&A, 50, 531

Kim D.-W., Elvis M., 1999, ApJ, 516, 9

Kim D., Im M., 2018, A\&A, 610, A31

Klindt L., Alexander D. M., Rosario D. J., Lusso E., Fotopoulou S., 2019, MNRAS, 488, 3109

Kratzer R. M., Richards G. T., 2015, AJ, 149, 61

Krishna G., Bhatnagar S., Wisotzki L., 1998, MNRAS, 299, L25

Lacy M., Canalizo G., Rawlings S., Sajina A., Storrie-Lombardi L., Armus L., Marleau F. R., Muzzin A., 2005, Mem. Soc. Astron. Italiana, 76, 154

Lake S. E., Wright E. L., Petty S., Assef R. J., Jarrett T. H., Stanford S. A., Stern D., Tsai C.-W., 2011, AJ, 143, 7

Laor A., Baldi R. D., Behar E., 2019, MNRAS, 482, 5513

Lu Y., Wang T., Zhou H., Wu J., 2007, AJ, 133, 1615

Mateos S., et al., 2012, MNRAS, 426, 3271

Mehdipour M., Costantini E., 2019, A\&A, 625, A25

Mingo B., et al., 2019, MNRAS, 488, 2701

Morabito L. K., et al., 2019, A\&A, 622, A15

O’Dea C. P., Baum S. A., Stanghellini C., 1991, ApJ, 380, 66

Panessa F., Baldi R. D., Laor A., Padovani P., Behar E., McHardy I., 2019, Nature, 3, 387 
Pâris I., et al., 2018, A\&A, 613, A51

Richards G. T., et al., 2003, AJ, 126, 1131

Rosario D. J., et al., 2020, MNRAS, in press

Schinnerer E., et al., 2007, ApJS, 172, 46

Schneider D. P., et al., 2010, AJ, 139, 2360

Serjeant S., Rawlings S., 1996, Nature, 379, 304

Siemiginowska A., Sobolewska M., Migliori G., Guainazzi M., Hardcastle M., Ostorero L., Stawarz Ł., 2016, ApJ, 823, 57

Smolčić V., et al., 2017a, A\&A, 602, A1

Smolčić et al., 2017b, A\&A, 602, A2

Stanghellini C., O’Dea C. P., Dallacasa D., Baum S. A., Fanti R., Fanti C., 1998, A\&AS, 131, 303

Stanley F., Harrison C. M., Alexander D. M., Swinbank A. M., Aird J. A., Del Moro A., Hickox R. C., Mullaney J. R., 2015, MNRAS, 453, 591

Stern D., et al., 2005, ApJ, 631, 163

Stern D., et al., 2012, ApJ, 753, 30

Urrutia T., Lacy M., Becker R. H., 2008, ApJ, 674, 80

Urrutia T., Becker R. H., White R. L., Glikman E., Lacy M., Hodge J., Gregg M. D., 2009, ApJ, 698, 1095

Urrutia T., Lacy M., Spoon H., Glikman E., Petric A., Schulz B., 2012, ApJ, 757, 125

Urry C. M., Padovani P., 1995, PASP, 107, 803

Webster R. L., Francis P. J., Petersont B. A., Drinkwater M. J., Masci F. J., 1995, Nature, 375, 469

White R. L., Helfand D. J., Becker R. H., Glikman E., de Vries W., 2007, ApJ, 654, 99

Whiting M. T., Webster R. L., Francis P. J., 2001, MNRAS, 323, 718

Wright E. L., et al., 2010, AJ, 140, 1868

York D. G., et al., 2000, AJ, 120, 1579

Zakamska N. L., Greene J. E., 2014, MNRAS, 442, 784

Zakamska N. L., et al., 2019, MNRAS, 489, 497

\section{APPENDIX A: eBOSS SOURCES IN S82}

For the final sample used in the analyses throughout this paper, the eBOSS-targeted QSOs were removed (see Section 2). This was due to the discrepancy in targeting of the two separate regions of Stripe 82 (often referred to as East; RA $\lesssim 36^{\circ}$ and West; RA $\gtrsim 330^{\circ}$ ), with eBOSS-targeted QSOs only present in the East field, which resulted in vastly different source densities (East: $159.5 \mathrm{deg}^{-2}$, West: $61.4 \mathrm{deg}^{-2}$ ). After removing these sources, this reduced the source density of the East field to $58.6 \mathrm{deg}^{-2}$, comparable to the West field and so the two regions could be combined for our analyses.

To check that the removal of these sources did not affect the main results, our analyses were repeated using the final sample, plus the 50 eBOSS-targeted QSOs from the East field. When including the eBOSS QSOs, the enhancement in the radio-detection fraction of rQSOs compared to cQSOs was still significant at a factor $\approx 2.6$ times higher for the rQSOs (compared to 3.3, see Section 3.1). The stacked radio-undetected colour-selected S82 rQSOs also appear brighter in the radio than the cQSOs for all redshifts. For the morphology analysis, there was still an enhancement in the faint, compact and extended categories for rQSOs of $4.0_{-0.7}^{+1.0}$, $1.9_{-0.4}^{+0.5}$ and $2.2_{-0.6}^{+1.1}$ respectively (compared to $4.2_{-0.9}^{+1.3}, 2.9_{-0.6}^{+0.9}$ and $2.2_{-0.8}^{+1.6}$ for the faint, compact and extended categories, respectively, see Section 3.3).

Removing or including the eBOSS-targeted QSOs therefore gives qualitatively the same results, which shows that our QSO selection is not driven by the SDSS targeting approach.

\section{APPENDIX B: SPURIOUS MATCHES FROM LARGER RADIUS MATCHING}

Fig. B1 displays the potential spurious matches that were not included in the final sample after matching with the larger $10^{\prime \prime}$ radius to search for FRII systems with a weak radio core. The thumbnails on the left show the four S82 sources and the thumbnails on the right show the one $\mathrm{C} 3 \mathrm{GHz}$ source. In each image a radio source is seen that is off-centre to the QSO position and does not appear to be associated with the QSO. For each source, a larger scale image was inspected to search for additional radio lobes that could be associated with a faint core but none were found.

\section{APPENDIX C: COMPARISON WITH FIRST}

To explore the effect of using higher resolution, deeper radio data to classify morphologies, our sample of S82 QSOs was cross-matched with the FIRST catalogue using a $10^{\prime \prime}$ search radius (false association rate of $\sim 0.3 \%$ ). This resulted in a sample of 99 QSOs (42 rQSOs and $57 \mathrm{cQSOs}$ ) down to the sensitivity limit of FIRST, but at 1.'8 resolution and higher SNR data given the greater sensitivity of S82. This analysis provides a more direct comparison to Klindt et al. (2019).

All extended and compact S82 QSOs were detected by FIRST, and only one FRII source had no FIRST detection at the optical QSO position ( $z=0.6 \mathrm{rQSO}$ ), due to the faint core (see Fig. C2). The remaining 65 S82 sources not detected by FIRST have S82 $1.4 \mathrm{GHz}$ fluxes that fall below $1 \mathrm{mJy}$. A total of 28 out of 99 of the matched sources were reclassified to a different morphology type: there were no faint or compact sources that were reclassified to an FRII-like morphology and no faint sources that were reclassified as extended with the higher resolution, deeper data; all of the faint sources were classified as compact.

Fig. C1 displays the morphology fractions for the S82-FIRST sample; the filled markers indicate the morphology classifications obtained from the S82 data (at 1.' 8 resolution), compared to the empty markers which are from the FIRST data $\left(5^{\prime \prime}\right.$ resolution). It is important to note that sources classified as faint in FIRST have $F_{\text {peak,FIRST }}<3 \mathrm{mJy}$, whereas for S82 they require $F_{\text {peak,S82 }}<1$ mJy. Similarly, compact in FIRST refers to sources with no extended emission beyond the $5^{\prime \prime}$ beam size compared to $1 . \prime 8$ in S82. In the FIRST data rQSOs have a preference for faint morphologies (in agreement with Klindt et al. 2019); however, due to the greater depth of S82 this difference subsides and boosts the red compact fraction. Due to the higher resolution of the $\mathrm{S} 82$ data, 6 rQSOs were reclassified from compact to extended. Since there were no extended rQSOs in the FIRST data, and no faint S82 sources that were reclassified as extended, this indicates that the enhancement in the extended category seen in Section 3.3 is driven mainly by the effect of higher resolution rather than the increased depth of the radio data. 
Spurious sources
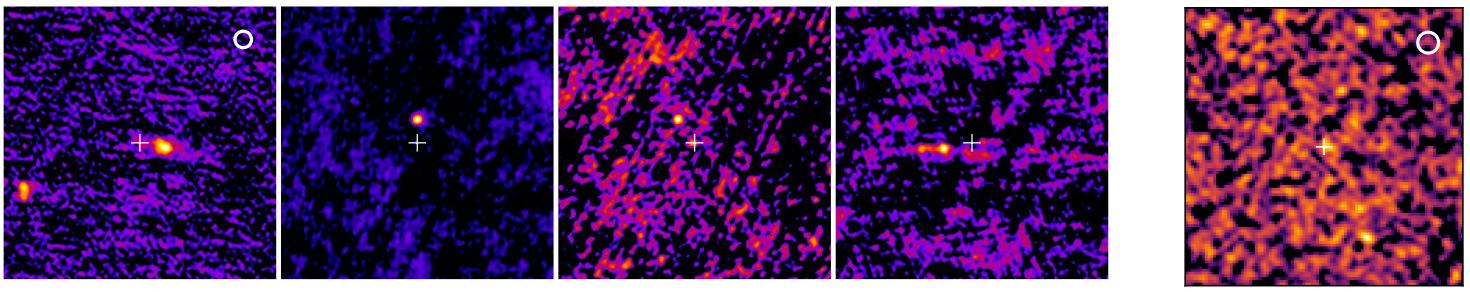

Figure B1. Thumbnails $\left(1^{\prime} \times 1^{\prime}\right)$ of potential S82 spurious matches (left), and a $20^{\prime \prime} \times 20^{\prime \prime}$ thumbnail of the potential C3GHz spurious match (right) found using the $10^{\prime \prime}$ search radius; these matches are not included in the final sample. The white cross indicates the optical QSO position and the circle on the first thumbnail displays the survey beam size (S82: 1".8; C3GHz: 0"75).

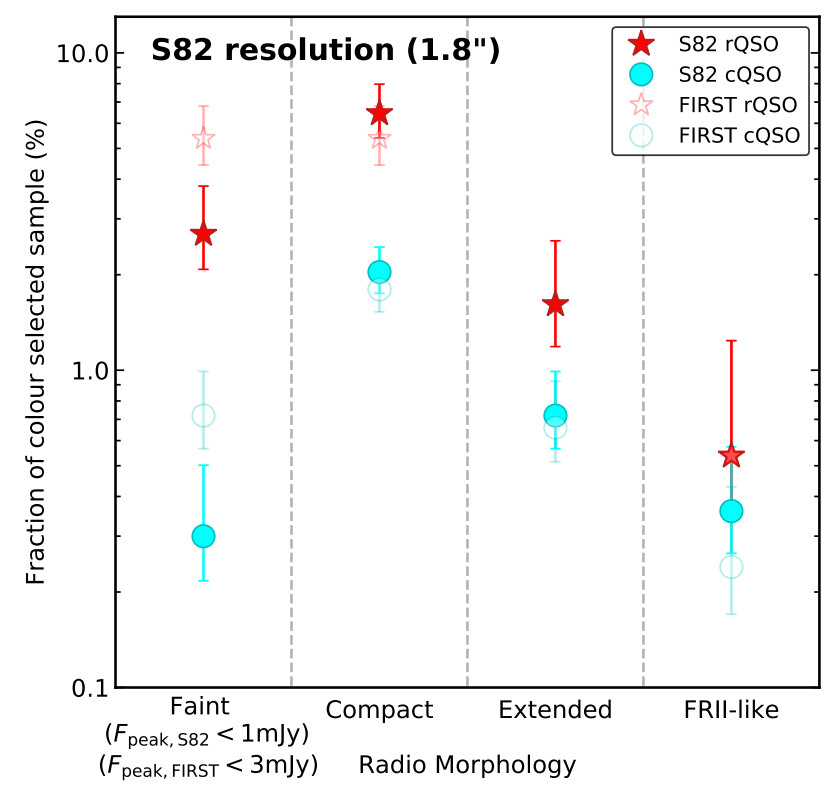

Figure C1. Radio morphology fractions of rQSOs and cQSOs for the S82FIRST matched subset ( 99 QSOs) at the sensitivity limit of FIRST. This plot indicates the impact of spatial resolution on our previous results with the FIRST data (Klindt et al. 2019). Filled and open markers indicate the morphologies obtained using the S82 (1".8 resolution) and FIRST (5" resolution) data, respectively. The red arrow gives a $3 \sigma$ upper limit for the FIRST extended rQSOs, due to that category containing no sources. The enhancement in the faint category for the FIRST data decreases when using the increased depth of the S82 data since we are now able to morphologically classify radio-detected QSOs with $1.4 \mathrm{GHz}$ flux densities of 1-3 mJy. Similarly, the extended category shows an enhancement for rQSOs in the S82 data due to the reclassification of FIRST compact sources in the higher resolution data (see Fig. 11).
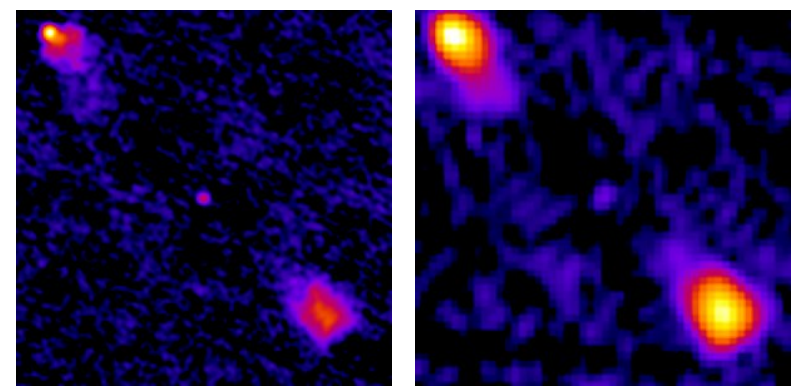

Figure C2. Thumbnails $(1.5 \times 1.5)$ of the one QSO classified as FRII-like in the S82 data not detected by FIRST at the SDSS QSO position due to the faint radio core. The left image displays the source in the S82 data and the right image displays the source in the FIRST data. 